\title{
Stochastic model of breakdown initiation in dielectric liquids
}

\author{
A L Kupershtokh ${ }^{1}$, E I Palchikov ${ }^{1}$, D I Karpov ${ }^{1}$, I Vitellas ${ }^{2}$, \\ D P Agoris ${ }^{3}$ and V P Charalambakos ${ }^{3}$ \\ ${ }^{1}$ Lavrentyev Institute of Hydrodynamics, Siberian Branch, Russian Academy of Sciences, \\ Lavrentyev prosp. 15, 630090 Novosibirsk, Russia \\ ${ }^{2}$ Testing Research \& Standards Center, Public Power Corp., 9 Leontarion, Kantza, 15351, \\ Pallini, Athens, Greece \\ ${ }^{3}$ University of Patras, GR 26500 Rion, Greece \\ E-mail: skn@hydro.nsc.ru
}

Received 13 June 2002

Published 18 November 2002

Online at stacks.iop.org/JPhysD/35/3106

\begin{abstract}
An electric strength of perfluorodibutyl ether and transformer oil in small gaps between the hemispherical electrodes was investigated experimentally under $\mathrm{AC}$ voltage of linearly increasing amplitude. The macroscopic stochastic approach was proposed that describes breakdown initiation by certain function $\mu(E)$. This function is the probability density of streamer inception on a small element of electrode area in a short interval of time and depends on local electric field. This macroscopic approach allows one to obtain the analytical expressions for breakdown initiation probability on various parameters of experiment for flat, cylindrical and hemispherical electrodes under constant DC test-voltage, DC and AC ramp test-voltages. Several methods were developed to reconstruct the function $\mu(E)$ using experimental data on series of breakdown voltages for case of breakdowns in small gaps between electrodes. The values of function $\mu(E)$ for transformer oil and perfluorodibutyl ether were determined in the range of electric field from 0.3 to $0.9 \mathrm{MV} \mathrm{cm}^{-1}$. It was shown that perfluorodibutyl ether is a prospective dielectric liquid, especially for use in environmentally appropriate technologies. Stochastic computer simulations of breakdown inception were carried out. The series of breakdown voltages and pitting on surface of hemispherical electrode were obtained using stochastic model.
\end{abstract}

\section{Nomenclature}

$a, b, h$ radiuses of inner and outer electrodes in cylindrical electrode geometry and length of work area of the electrodes

$A$

$a(\beta)$

$b, b_{*}$

mental parameters

C constant

$d \quad$ gap length

$E \quad$ electric field strength

$E_{0}^{*} \quad$ amplitude value of mean electric field strength along the symmetry axis of two hemispherical electrodes that corresponds to value of $V_{\mathrm{EFF}}^{*}$ $\left\langle E_{0}\right\rangle \quad$ average value of amplitude of effective electric field of breakdown

$f(E)$ probability density of breakdown initiation in short time interval

$F(n)$ dimensionless function of index $n$

$g \quad$ parameter in approximations of the function $\mu(E)$

$H(t)$ reduced statistical time lag of breakdown (integral of electric-field action)

$k \quad$ rate of increase of amplitude value of voltage

$k_{\mathrm{e}} \quad$ rate of increase of effective value of applied voltage

$n$ index in power-law approximation of the function $\mu(E)$

$N_{0} \quad$ the total number of breakdowns in a series

$N_{i} \quad$ number of breakdowns in a series of breakdown that have occurred not earlier than $i$ th half-cycle of $\mathrm{AC}$ voltage 
$P_{+} \quad$ probability of electrical breakdown

$P_{l} \quad$ Legendre polynomial of index $l$

$R \quad$ electrode radius

$S \quad$ electrode area

$S_{*} \quad$ effective area of electrode

$t \quad$ time

$t_{\mathrm{S}},\left\langle t_{\mathrm{S}}\right\rangle \quad$ statistical time lag and its mean value for a series of breakdowns

$V \quad$ applied voltage

$\left\langle V_{\mathrm{EFF}}\right\rangle \quad$ average effective value of breakdown voltage

$V_{\mathrm{EFF}}^{*} \quad$ effective value of voltage in a series at which breakdown occurs with fixed probability 0.63

$x, y \quad$ coordinates

$\alpha \quad$ Azimuthal angle in bispherical coordinates

$\beta \quad$ reduced gap length

$\gamma \quad$ coefficient of admissible reducing of electric field strength along the surface of hemispherical in effective area of electrode

$\Gamma \quad$ gamma-function

$\delta \quad$ nominal roughness of electrode surface

$\Delta E_{l} \quad$ increment of electric field strength for $l$ half-cycles of applied voltage

$\Delta \quad$ relative maximum difference between average breakdown voltages in experimetns and in simulations

$\varepsilon \quad$ permittivity

$\theta \quad$ polar angle on a sphere

$\mu(E) \quad$ probability density of breakdown initiation from small electrode area in short time interval

$v, m \quad$ exponents in functions of surface roughness

$\xi, \eta \quad$ bispherical coordinates

$\sigma \quad$ standard deviation of breakdown voltages for series of breakdown

$\omega \quad$ cyclic frequency of AC voltage

$\varphi \quad$ electric field potential

\section{Introduction}

It is usually implied that the ability of a dielectric to maintain the dielectric properties under the action of strong electric fields is characterized by its electric strength. However, it is well-known, that the average value of electric field, at which breakdown of a dielectric occurs, depends also on specific experimental conditions such as the form and the sizes of electrodes, the distance between them, the magnitude and the form of applied voltage, etc [1-6]. Therefore, the classical concept about fixed 'electric strength' fails. Instead of this, the concept of 'dynamic electric strength' of dielectric that depends on the specific conditions listed above has to be used. Well-known time-voltage curves are the particular features of dynamic electric strength.

Moreover, it is well-known that the pre-breakdown processes in liquid dielectrics have a stochastic nature. Numerous experimental data point to the principal role of stochastic processes at a breakdown in dielectric liquids (e.g. statistical time lag, asymmetry and non-reproducibility of detailed streamer structure, tooth-like shape of recordings of current and light pulses, etc). Thus, an adequate description of stochastic regularities of dielectric breakdown has to include probability distribution functions for such processes.
One of the stochastic processes is the initiation of streamer due to the development of a series of microscopic phenomena at the electrode surface and in a thin dielectric layer contiguous to it. The duration of this stage of breakdown (called the statistical time lag $t_{\mathrm{S}}$ ) is a random variable for which the probability density depends on the electric field and its distribution along the surface of the electrodes. This stage of breakdown determines a dynamic electric strength.

Many authors made efforts to describe stochastic regularities of breakdown using various statistical distributions. The attempts to apply statistics of extreme values [1] and Weibull's distributions [5-12] for interpretation of the numerous available experimental data are well-known. Unfortunately, these approaches do not allow one to describe in a simple way how the complete set of experimental conditions (duration and waveform of applied voltage, form and size of electrodes, etc) influences the breakdown.

One of the attempts to investigate the essentially stochastic character of prebreakdown processes in dielectric liquids was made by Lewis in [2]. He proposed to use the distribution function in which the reciprocal value of mean statistical time $\operatorname{lag} f(E)$ was used. The last dependence means the probability density of breakdown initiation in a short time interval.

In the last few years many researchers showed that the probability of breakdown initiation in some local area of dielectric should depend on magnitude of local electric field in this area and on properties of the dielectric liquid and of electrode surface, provided that pressure and temperature are constant during experiment. The dielectric strength of liquid dielectrics strongly depends on the presence of weak links at the surface of electrodes such as electrodes roughness, free conducting particles or gas micro-bubbles. This fact was proved in experiments where the thin conducting 'diffusion' layers on the surface of both electrodes were produced [13]. The electric strength of water increased approximately by fourfold. This circumstance allows us to make a conclusion that the main contribution was made by the surface effect but not by the volume effect.

These features of breakdown phenomenon allowed us to describe the dynamic electric strength of specific dielectric quantitatively, taking into account the essentially stochastic nature of breakdown. In [14-17] it was proposed that the basic stochastic processes of streamer inception at the electrode could be described by macroscopic function $\mu(\mathrm{E})$. This function is the probability density of breakdown initiation in a short time interval at a small element of an electrode surface near which the electric-field value equals $E$.

Specific form of function $\mu(E)$ is closely related to the physical nature of microscopic processes occurring at the surface of electrodes. Several mechanisms of breakdown initiation are known such as thermal-electric ionization and dissociation, dissociative ionization, impact ionization, tunnel effect, bubble mechanism of breakdown, etc. For example, for mechanisms of thermal-electric ionization and dissociation, the dependence of $\mu(E)$ on electric field is proportional to exponential function. This kind of dependence $f(E)=\exp (\lambda E-B)$ was used, for example, in [2] for approximation of experimental data on the probability density of a breakdown in $n$-hexane for rectangular voltage pulses.

Usually, parallel competition between several mechanisms takes place under different conditions. For example, 
three mechanisms of breakdown initiation (bubble-like mechanisms of breakdown both from the cathode and from the anode and the ionization mechanism of breakdown from the anode) compete at the breakdowns in $n$-hexane in strong electric fields $E_{0}>1 \mathrm{MV} \mathrm{cm}^{-1}$ in the time range from 10 to $1000 \mathrm{~ns}$ under normal pressure $[18,19]$. Moreover, the dielectric strength of liquid dielectrics strongly depends on presence of weak links at the surface of electrodes such as electrodes roughness, free conducting particles or gaseous micro-bubbles and also on state of surface (chemical layers, adsorption phenomena etc).

It is quite reasonable in this complex case to approximate the function $\mu(E)$ by some formula of general form that does not relate to specific physical process. All available experimental data point out that the function $\mu(E)$ is monotonous one. One can use any kind of monotonically increasing functions for approximating $\mu(E)$, but this approximate dependence should increase sharply with increase in the electric field to describe the wellknown 'quasi-threshold' character of breakdown phenomena. Simplest formulae that satisfied these requirements are powerlaw and exponential functions. The parameters of the function $\mu(E)$ depend on the properties of a specific dielectric and on the properties of the electrodes.

The conception of dynamic electric strength originates from stochastic approach by averaging the breakdown voltages over the probability distributions. For a specific dielectric, the function $\mu(E)$ defines directly the dynamic electric strength for every specific condition. It is obvious that function introduced in [2] $f(E)=\int_{S} \mu(E) \mathrm{d} s$, where the domain of integration is the entire electrode area.

Our macroscopic approach [14-17, 20-22] allows one to obtain the dependencies of the breakdown initiation probability in time on the applied voltage, its waveform, electrode area, gap length, etc without analysing the physical mechanisms of breakdown initiation at microscopic level and to simulate the breakdown, including its stochastic features. Vice versa, it is possible to reconstruct the probability density function $\mu(E)$ from experimental data.

Thus, the macroscopic approach involves:

- Accumulation of experimental data on stochastic features of breakdown inception in dielectric liquids.

- Theoretical description of stochastic features of breakdown inception.

- Reconstruction of macroscopic function $\mu(E)$ for specific dielectric liquids, using the experimental data on breakdown.

- Development of a physical model, that by means of computer modelling describes breakdown inception in dielectric liquids and also main stochastic effects, arising at initial stage of streamer inception.

Clearly, our macroscopic approach is applicable to breakdown initiation that is governed by area effect. At present, the existing experimental data do not give complete understanding where the area effect is dominating. Our macroscopic approach is operative at least for breakdown initiation in small gaps (of the order of millimetres or less) where characteristic sizes of stressed electrode area are much greater than gap length.

In this paper the stochastic theory of inception of breakdown in liquid dielectrics under constant DC, ramp DC and linearly increasing AC voltages was developed. This macroscopic approach was applied to the data on breakdown in transformer oil and perfluorodibutyl ether in the case of linearly increasing AC voltage and hemispherical electrodes.

Perfluorocarbon liquids are well-known as good dielectrics with high resistance and electric strength [23-26]. Besides, they have very small viscosity and high density. The most important features are their chemical inertness and their non-flammability. Considered together, these properties make perfluorocarbon liquids suitable for industrial application in high-voltage equipment.

\section{Experiments}

Experiments on breakdown in synthetic transformer oil 'TECHNOL 2002 (ISO 9001)' were carried out. A new pair of polished spherical stainless steel electrodes with surface radius $R=19 \mathrm{~mm}$ and new portion of dielectric liquid were used in each series of experiments. All experiments were carried out with the same conditions of electrode surface taking into account the importance of surface state mentioned above. The gap lengths between the electrodes $d$ were in the range from 0.5 to $2.5 \mathrm{~mm}$. High voltage tests were carried out using the standard generator 'Baur A-6832'. The amplitude of AC voltage of frequency $50 \mathrm{~Hz}$ increased with a constant rate. The ambient temperature was $25^{\circ} \mathrm{C}$.

In experiments, the current effective value of voltage $V_{\mathrm{EFF}}$ at which breakdown of a dielectric occurred was registered (figure 1). A built-in electronic device removed the voltage from the electrodes immediately after breakdown. The rate

(a)
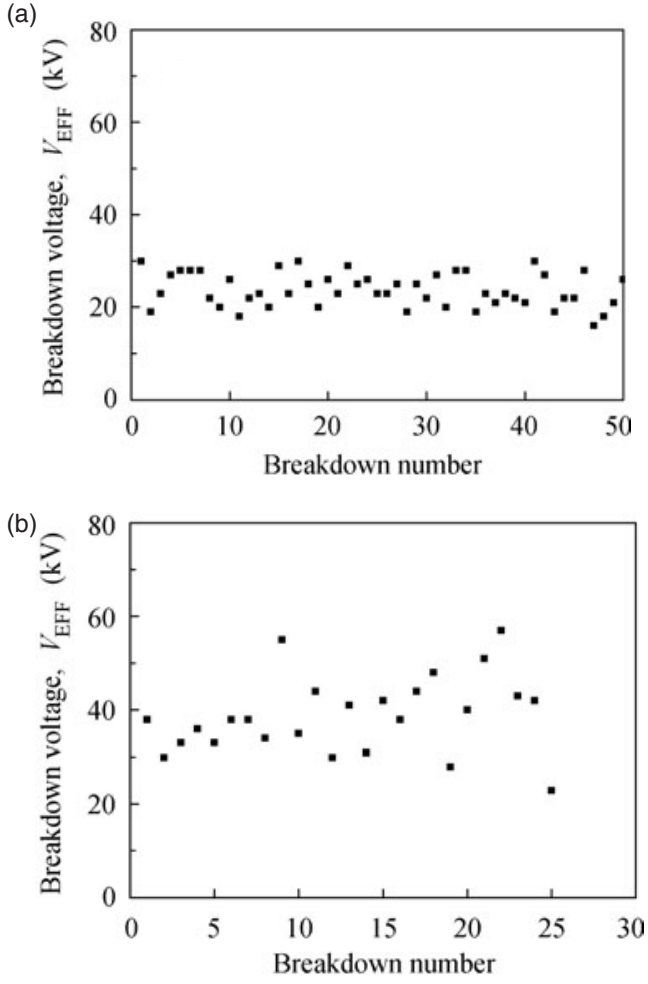

Figure 1. Typical series of breakdowns in transformer oil under AC voltage of linearly increasing amplitude. (a) $d=0.5 \mathrm{~mm}$, $k_{\mathrm{e}}=3 \mathrm{kV} \mathrm{s}^{-1}$. (b) $d=1.66 \mathrm{~mm}, k_{\mathrm{e}}=1 \mathrm{kV} \mathrm{s}^{-1}$. 
Table 1. Experiments on breakdown in transformer oil.

\begin{tabular}{|c|c|c|c|c|c|c|c|}
\hline No & $\begin{array}{l}d \\
(\mathrm{~mm})\end{array}$ & $\begin{array}{l}k_{\mathrm{e}} \\
\left(\mathrm{kV} \mathrm{s}^{-1}\right)\end{array}$ & $N_{0}$ & $\begin{array}{l}\left\langle V_{\mathrm{EFF}}\right\rangle \\
(\mathrm{kV})\end{array}$ & $\begin{array}{l}\left\langle E_{0}\right\rangle \\
\left(\mathrm{kV} \mathrm{cm}^{-1}\right)\end{array}$ & $\begin{array}{l}V_{\mathrm{EFF}}^{*} \\
(\mathrm{kV})\end{array}$ & $\begin{array}{l}E_{0}^{*} \\
\left(\mathrm{kV} \mathrm{cm}^{-1}\right)\end{array}$ \\
\hline \multirow[t]{3}{*}{1} & 2.5 & 0.5 & 60 & 50.6 & 286 & 53 & 300 \\
\hline & 2.5 & 1 & 60 & 55.5 & 314 & 58 & 328 \\
\hline & 2.5 & 3 & 60 & 64.0 & 362 & 71 & 402 \\
\hline \multirow[t]{3}{*}{2} & 1.0 & 0.5 & 40 & 24.1 & 341 & 25 & 354 \\
\hline & 1.0 & 1 & 40 & 24.6 & 348 & 25.5 & 361 \\
\hline & 1.0 & 3 & 39 & 29.8 & 421 & 34 & 481 \\
\hline \multirow[t]{3}{*}{3} & 0.5 & 0.5 & 48 & 20.5 & 580 & 21.5 & 608 \\
\hline & 0.5 & 1 & 50 & 22.4 & 634 & 24 & 679 \\
\hline & 0.5 & 3 & 50 & 23.8 & 673 & 25 & 707 \\
\hline \multirow[t]{3}{*}{4} & 0.83 & 0.5 & 27 & 25.7 & 438 & 30 & 511 \\
\hline & 0.83 & 1 & 27 & 29.1 & 496 & 31 & 528 \\
\hline & 0.83 & 3 & 27 & 27.6 & 470 & 30.5 & 520 \\
\hline \multirow[t]{3}{*}{5} & 1.66 & 0.5 & 25 & 32.6 & 278 & 36 & 307 \\
\hline & 1.66 & 1 & 25 & 38.9 & 331 & 42 & 358 \\
\hline & 1.66 & 3 & 25 & 45.8 & 390 & 49 & 417 \\
\hline \multirow[t]{3}{*}{6} & 2.5 & 0.5 & 25 & 42.2 & 238 & 46 & 260 \\
\hline & 2.5 & 1 & 25 & 46.7 & 264 & 49 & 277 \\
\hline & 2.5 & 3 & 25 & 57.0 & 322 & 61 & 345 \\
\hline
\end{tabular}

of increase of effective value of applied voltage $k_{\mathrm{e}}$ was changed cyclically after each breakdown in special order 0.5 , $1,3,0.5,3,1 \mathrm{kV} \mathrm{s}^{-1}$. Thus, three data sets of breakdown voltages were obtained in one series of experiments under identical conditions. The period between breakdowns was approximately $3 \mathrm{~min}$. The conditioning effect was observed in every series of breakdowns. We took into account only the breakdowns after 15 or 20 shots in series. The results of six series of breakdowns are shown in table 1 .

Here, No is the series number, $N_{0}$ is the number of breakdowns, $\left\langle V_{\mathrm{EFF}}\right\rangle$ is the average effective value of breakdown voltage, $\left\langle E_{0}\right\rangle$ is the corresponding value of amplitude of electric field averaged along the axis between electrodes, $V_{\mathrm{EFF}}^{*}$ is the effective value of a voltage at which in a series of experiments breakdown occurred with the fixed probability $P_{+}(t)=0.63$, $E_{0}^{*}$ is the corresponding amplitude value of electric field averaged along the axis of electrode system.

Two typical distributions of places of breakdown initiation on the surfaces of the electrodes corresponding to the series 4 and 6 are shown in figure 2 (breakdown pitting). The total numbers of breakdown events are equal to 126 in the series 4 and 120 in the series 6 . It is clearly seen that characteristic size of pitting region increases with the gap spacing.

The experiments on breakdown in the perfluorodibutyl ether $\mathrm{CF}_{3}-\left(\mathrm{CF}_{2}\right)_{3}-\mathrm{O}-\left(\mathrm{CF}_{2}\right)_{3}-\mathrm{CF}_{3}$ were also carried out. This liquid is colourless and odorless with density $1.7 \mathrm{~g} \mathrm{~cm}^{-3}$ and permittivity $\varepsilon=1.82$. New portion of dielectric liquid were used in each series of experiments. The liquid was previously boiled for degassing over a period of $1-2 \mathrm{~h}$ at the temperature of $101^{\circ} \mathrm{C}$ with a backflow condenser to prevent boiling out of liquid. Then the liquid was filtered to avoid the effect of contamination. The effective value of the AC voltage increased with a constant rate $k_{\mathrm{e}}=2 \mathrm{kV} \mathrm{s}^{-1}$. The frequency was $50 \mathrm{~Hz}$. During the experiments, the current effective value of voltage $V_{\mathrm{EFF}}$ at which breakdown of a dielectric occurred was registered (figure 3(a)). Typical bar chart for breakdown voltages corresponding to figure $3(a)$ is shown in figure 4. The surfaces of hemispherical stainless steel and brass electrodes were polished before each series of
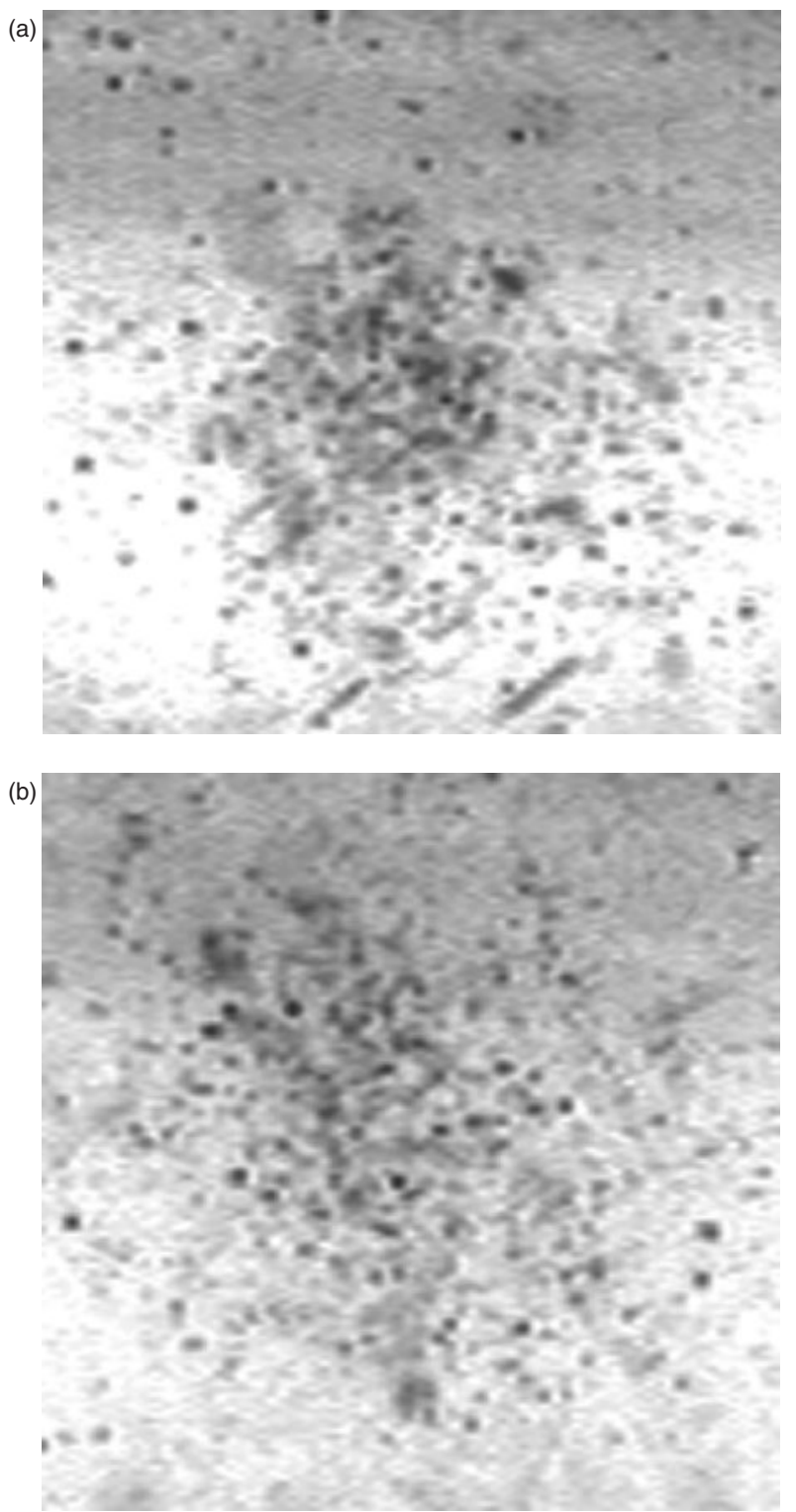

Figure 2. Photos of the pitting on surface of the stainless steel electrodes after series of breakdown in transformer oil. Areas of size $6 \times 6 \mathrm{~mm}$ are shown and $R=19 \mathrm{~mm} . d=0.83 \mathrm{~mm}(a)$ and $2.5 \mathrm{~mm}(b)$

experiments (approximately 100 breakdowns in each series). The procedure of polishing for every electrode was uniform to ensure the same nominal roughness taking into account the importance of surface state mentioned above. The results of eight series of breakdowns in perfluorodibutyl ether are presented in table 2 .

In all series of breakdowns, one can see a rather significant statistical difference in breakdown voltages (figures 1, 3(a) and 4). This is a direct consequence of the stochastic regularities of the process.

\section{Calculation of the electric-field distribution between hemispherical electrodes}

A good approximation of the distribution of electric field strength on the surface of hemispherical electrodes is given 
(a)

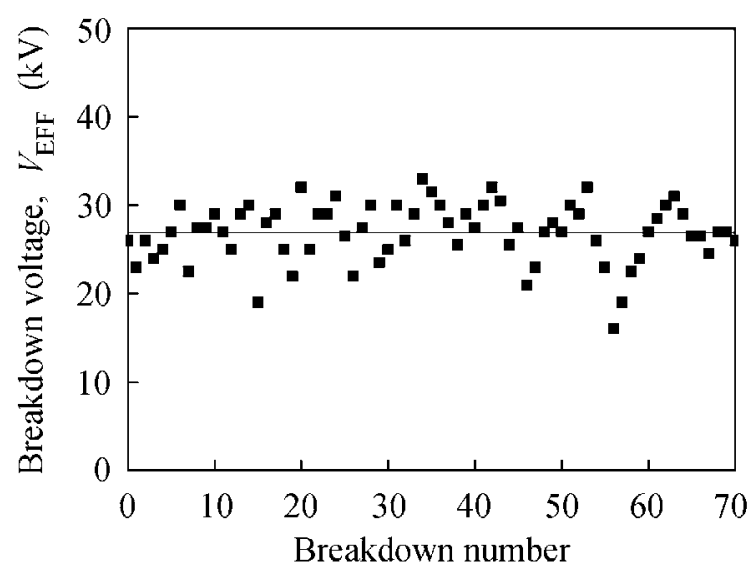

(b)

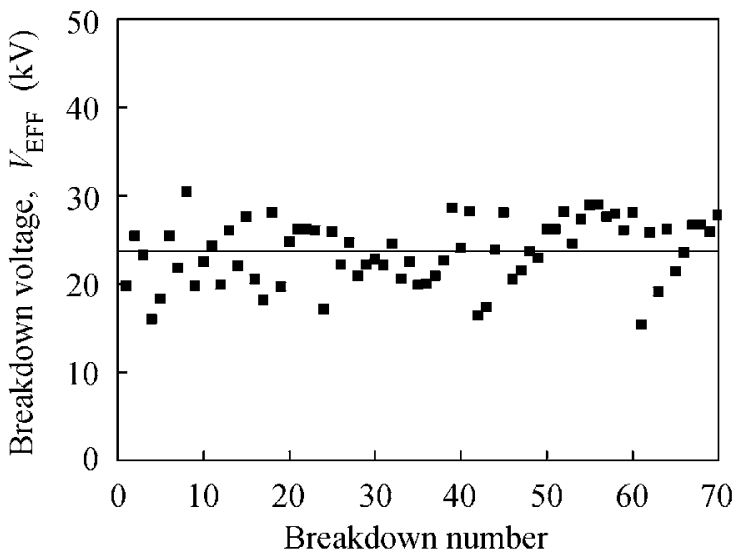

Figure 3. Typical series of breakdowns in perfluorodibutyl ether under AC voltage of linearly increasing amplitude. Experiments $(a)$ and computer simulations $(b)$. Stainless steel electrodes of radius $R=30 \mathrm{~mm} . d=0.44 \mathrm{~mm} . N_{0}=71$.

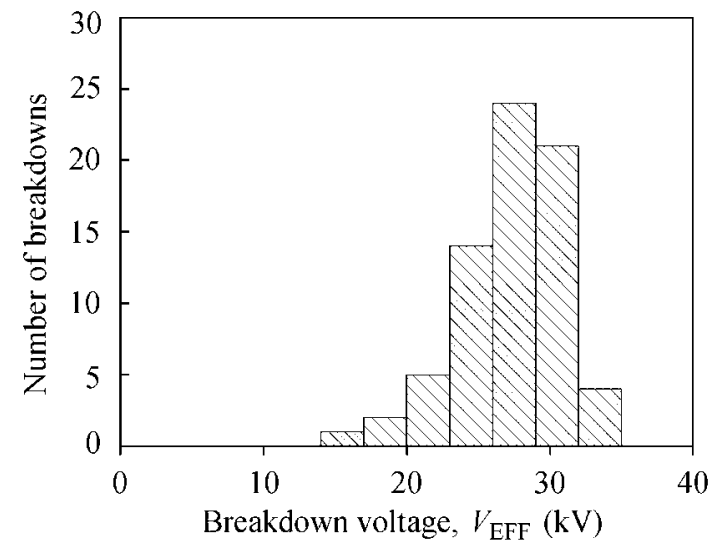

Figure 4. Typical bar chart of breakdown voltages in perfluorodibutyl ether under AC voltage of linearly increasing amplitude. Stainless steel electrodes of radius $R=30 \mathrm{~mm}$. $d=0.44 \mathrm{~mm}$.

by field distribution in the gap between two metallic spheres (figure 5). Here $V$ is the applied voltage, $R$ is the radius of spherical electrodes, and $d$ is the gap length between them. The electric-field distribution in the region between electrodes
Table 2. Experiments on breakdown in perfluorodibutyl ether.

\begin{tabular}{llrllll}
\hline $\begin{array}{l}d \\
(\mathrm{~mm})\end{array}$ & $\begin{array}{l}R \\
(\mathrm{~mm})\end{array}$ & $N_{0}$ & $\begin{array}{l}\left\langle V_{\mathrm{EFF}}\right\rangle \\
(\mathrm{kV})\end{array}$ & $\begin{array}{l}\left\langle E_{0}\right\rangle \\
\left(\mathrm{kV} \mathrm{cm}^{-1}\right)\end{array}$ & $\begin{array}{l}V_{\mathrm{EFF}}^{*} \\
(\mathrm{kV})\end{array}$ & $\begin{array}{l}E_{0}^{*} \\
\left(\mathrm{kV} \mathrm{cm} \mathrm{cm}^{-1}\right)\end{array}$ \\
\hline \multicolumn{7}{c}{ Stainless steel electrodes } \\
0.44 & 30 & 71 & 26.9 & 865 & 28.0 & 900 \\
0.9 & 30 & 101 & 41.2 & 647 & 43.5 & 684 \\
1.7 & 30 & 115 & 50.5 & 420 & 54.5 & 453 \\
2.5 & 30 & 120 & 70.8 & 400 & 75.0 & 424 \\
0.44 & 40 & 161 & 20.3 & 652 & 23.0 & 740 \\
0.9 & 40 & 135 & 37.7 & 592 & 42.0 & 660 \\
1.7 & 40 & 130 & 49.4 & 411 & 52.0 & 433 \\
2.5 & 40 & 80 & 73.4 & 415 & 77.0 & 436 \\
\hline
\end{tabular}

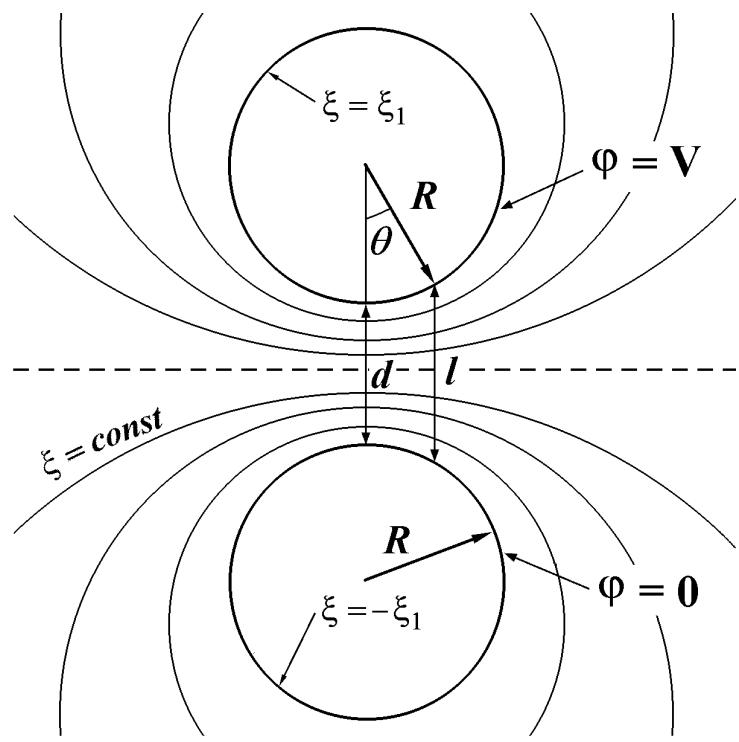

Figure 5. The configuration of spherical electrodes and the surfaces of equal electric field potential $\xi=$ const.

was obtained analytically by solving the Laplace equation in bispherical coordinates

$$
\begin{aligned}
E(\xi, \eta)= & \frac{E_{0} d \sqrt{2(\cosh \xi-\cos \eta)}}{2 R \sinh \xi_{1}} \\
& \times \sum_{l=0}^{\infty} \frac{\exp \left(-(l+1 / 2) \xi_{1}\right) P_{l}(\cos \eta)}{\sinh \left((2 l+1) \xi_{1}\right)} \\
& \times\left\{\sinh \left(\left(l+\frac{1}{2}\right)\left(\xi+\xi_{1}\right)\right) \sinh \xi\right. \\
& +2(\cosh \xi-\cos \eta)\left(l+\frac{1}{2}\right) \\
& \left.\times \cosh \left(\left(l+\frac{1}{2}\right)\left(\xi+\xi_{1}\right)\right)\right\} .
\end{aligned}
$$

Here $E_{0}=V / d$ is the average electric field strength along an axis between electrodes, $\xi$ and $\eta$ are bispherical coordinates (electric field potential and electric strength do not depend on 


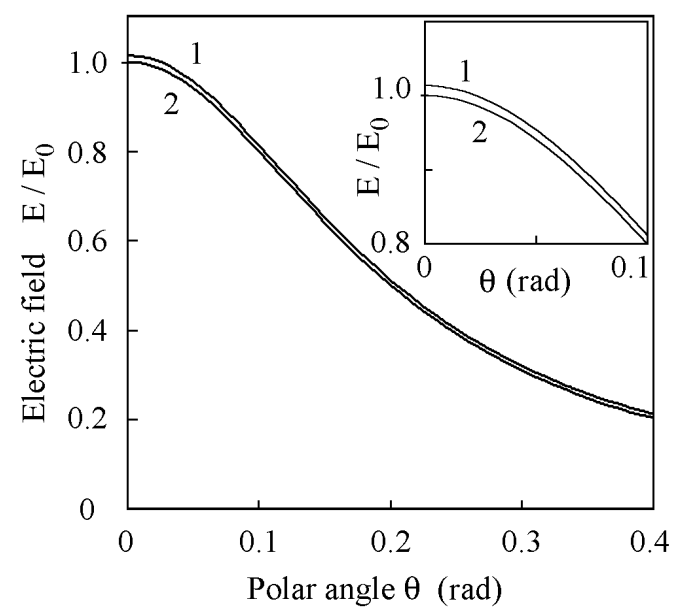

Figure 6. Electric-field distribution along the surface of a spherical electrode for $\beta=0.02$. Exact solution (1) is shown by curve 1 and approximate solution (3) is shown by curve 2 .

azimuthal angle $\alpha$ because of symmetry of the problem, $\left.-\xi_{1}<\xi<\xi_{1}, 0<\eta<\pi\right), \mathrm{P}_{l}$ is the Legendre polynomial of index $\left.l, \xi_{1}=\ln (1+\beta+\sqrt{\beta(2+\beta})\right), \beta=d / 2 R$ is the reduced length of the gap between identical electrodes. The relation between bispherical coordinate $\eta$ and polar angle $\theta$ on the sphere counted from the symmetry axis is given by the expression

$$
\cos \eta=\frac{1-(1+\beta) \cos \theta}{1+\beta-\cos \theta} .
$$

For a quasi-uniform field in a narrow gap between spherical electrodes, the electric field strength changes weakly along electric field lines. Therefore, one can consider that $E \approx V / l$ (see figure 5) and the following approximate formula for electric-field distribution at the surface of electrode near the symmetry axis is valid $[15,17]$

$$
E \approx \frac{E_{0}}{1+(1-\cos \theta) / \beta} .
$$

The plot of a relative electric field $E / E_{0}$ at electrode surface versus $\theta$ is given in a figure 6 . The direction $\theta=0$ corresponds to the maximum value of electric field at the apex. Only a small part of the electrode area near the symmetry axis makes a major contribution to breakdown inception because of the sharp dependence of the function $\mu(E)$ on the electric field. For this region, the approximate formula (3) (figure 6, curve 2) practically coincides with exact solution (1) (figure 6, curve 1). For example, the difference between them is less than $2 \%$ of the maximum field strength for a gap distance corresponding up to $\beta=0.02$ [17].

The distribution of electric field strength along the symmetry axis of system of two spherical electrodes is shown in figure 7. The electric field is not constant along the electric field lines and the maximal value on the electrode surface is somewhat higher than the average value along the axis of symmetry $E_{0}$. The coordinate $z$ along the symmetry axis is expressed through bispherical coordinate $\xi$ as $z=$ $\left(R \sinh \xi_{1} \sinh \xi\right) /(\cosh \xi+1)$. Factor of amplification of electric field on the surface of electrode in comparison with the average value along the symmetry axis $a(\beta)=E_{\max } / E_{0}$

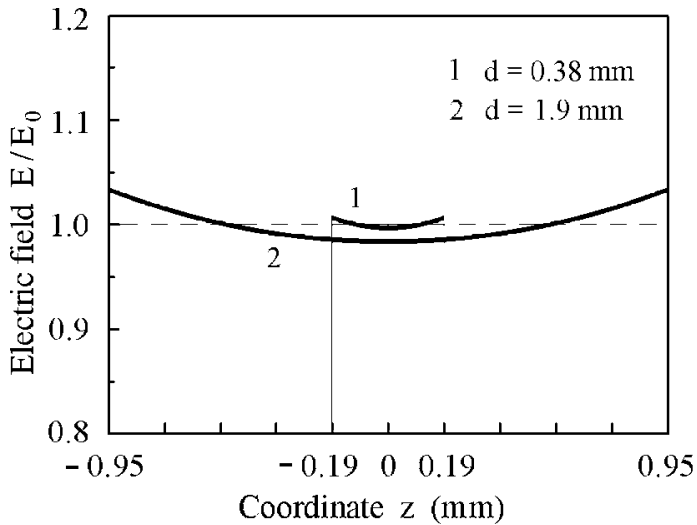

Figure 7. Distributions of electric field strength along the symmetry axis of two spherical electrodes obtained from the exact solution (1) at $\beta=0.01$ (curve 1 ) and $\beta=0.05$ (curve 2). $R=19 \mathrm{~mm}$

Table 3. Correction factor $a(\beta)$.

\begin{tabular}{llllll}
\hline$\beta$ & 0.01 & 0.05 & 0.066 & 0.1 & 0.2 \\
$a(\beta)$ & 1.007 & 1.034 & 1.044 & 1.068 & 1.13 \\
\hline
\end{tabular}

depends only on the parameter $\beta$ (table 3 ). The data of this table can be approximated by the formula $a=1+0.666 \beta$ with very good accuracy. Use of correction factor $a(\beta)$ allows one to extend considerably the range of the applicability of formula (3) to the values $\beta \approx 0.1$, using a product $a(\beta) E_{0}$ instead of $E_{0}$.

\section{Macroscopic approach to breakdown initiation}

\subsection{Probability of breakdown initiation}

In stochastic approach proposed earlier in [14-17, 20-22], macroscopic function $\mu(E)$ was introduced which depends on local electric field. The function $\mu(E)$ has the physical sense of probability density of breakdown initiation on a small element of electrode surface in a short interval of time. It was supposed that probability of breakdown inception near small element of surface of electrode at time $t$ does not depend on previous moments of time and on events near other elements of electrode $[15,16]$ (Poisson process). In this case, the probability of breakdown inception in time $t$ is equal to

$$
P_{+}(t)=1-\exp (-H)
$$

where the value of integral of electric-field action $H(t)$ is expressed through the function $\mu(E)$ and changes in time as

$$
H(t)=\int_{0}^{t}\left(\int_{S} \mu(E) \mathrm{d} s\right) \mathrm{d} t .
$$

In the case of applied voltage $V=$ const., the statistical time lag of breakdown $t_{\mathrm{S}}$ is exponentially distributed. The probability density function takes the form

$$
f\left(t_{\mathrm{S}}\right)=\left(\int_{S} \mu(E) \mathrm{d} s\right) \exp \left(-t_{\mathrm{S}} \int_{S} \mu(E) \mathrm{d} s\right) .
$$


For flat electrodes we have

$$
\begin{gathered}
H(t)=S \int_{0}^{t} \mu(E) \mathrm{d} t, \\
P_{+}(t)=1-\exp \left(-S \int_{0}^{t} \mu(E) \mathrm{d} t\right),
\end{gathered}
$$

where $S$ is the area of the electrode. In the simplest case of constant DC test-voltage, the analytical expressions for the integral of electric-field action $H(t)$ were derived from (7) for flat electrodes

$$
H(t)=S \mu(E) t .
$$

From the distribution (6) of random variable $t_{\mathrm{S}}$, one can easy obtain an expression for determination of the value of function $\mu(E)$

$$
\mu(E)=\frac{1}{S\left\langle t_{\mathrm{S}}\right\rangle},
$$

where $\left\langle t_{\mathrm{S}}\right\rangle$ is the mean value of statistical time lag measured in experiments.

For hemispherical electrodes with a small gap distance between them, it is possible to turn the integration in (5) from integral over the surface of electrode to the integral over electric field, using approximation (3) $[15,17]$

$$
\int_{S} \mu(E) \mathrm{d} s \approx d \pi R E_{0} \int_{0}^{E_{0}} \frac{\mu(E)}{E^{2}} \mathrm{~d} E .
$$

On the right-hand side of equation (11) we used zero as lower limit of integration, bearing in mind the extremely sharp dependence of function $\mu(E)$ on electric field.

In general case of arbitrary form of function $\mu(E)$ for hemispherical electrodes we have

$$
\begin{gathered}
H(t)=d \pi R \int_{0}^{t}\left(E_{0} \int_{0}^{E_{0}} \frac{\mu(E)}{E^{2}} \mathrm{~d} E\right) \mathrm{d} t, \\
P_{+}=1-\exp \left(-d \pi R \int_{0}^{t}\left(E_{0} \int_{0}^{E_{0}} \frac{\mu(E)}{E^{2}} \mathrm{~d} E\right) \mathrm{d} t\right),
\end{gathered}
$$

where $E_{0}=V(t) / d$.

Using (11), we introduced in [15] the concept of the effective area for hemispherical electrodes for small gaps in accordance with the following formula:

$$
S_{*}=\frac{d \pi R E_{0} \int_{0}^{E_{0}}\left(\mu(E) / E^{2}\right) \mathrm{d} E}{\mu\left(E_{0}\right)} .
$$

It means that any probabilities $P_{+}(E)$ plotted for flat electrodes could be used also for hemispherical electrodes in the case of narrow gaps $(\beta<0.1)$ if instead the value $S$ we imply the effective area $S_{*}$.

For example, for hemispherical electrodes from (8) and (14) one can obtain the expression for probability of breakdown under constant test-voltage pulse in the form

$$
\begin{aligned}
P_{+}(t) & =1-\exp \left(-t S_{*} \mu\left(E_{0}\right)\right) \\
& =1-\exp \left(-t d \pi R E_{0} \int_{0}^{E_{0}} \frac{\mu(E)}{E^{2}} \mathrm{~d} E\right) .
\end{aligned}
$$

For DC ramp test-voltage $V=k t$ and for AC ramp test-voltage $V=k t \sin (\omega t)$, for which voltage amplitude increasing in time as $V_{\mathrm{A}}=k t$, it is possible to turn the integration in (8) and (13) from integral over time to the integral over electric field. Here $\omega$ is the frequency of AC voltage. In this case, one can obtain simple analytical expressions for probability of breakdown inception $P_{+}(E)$.

\subsection{Power-law approximation of $\mu(E)$}

The power-law dependence

$$
\mu(E)=A\left(\frac{E}{E_{1}}\right)^{n}
$$

is the simplest approximation of function $\mu(E)$ that satisfied the requirements mentioned above. If the function $\mu(E)$ for a specific dielectric can be approximated in a certain range by formula (16), the analytical expressions for integral of electricfield action $H(t)$ can be derived from (7) [20-22] for flat electrodes

$$
H(t)=\frac{S A}{E_{1}^{n}} \int_{0}^{t} E^{n} \mathrm{~d} t
$$

and from (12) for hemispherical electrodes

$$
H(t)=\frac{d \pi R A}{(n-1) E_{1}^{n}} \int_{0}^{t} E_{0}^{n} \mathrm{~d} t .
$$

In (18), the value of effective area for hemispherical electrodes is $S_{*}=\pi R d /(n-1)$. The effect of an increase in the area, on which the breakdown originated, with an increase in the product of electrode radius and gap spacing $R d$ is well-known from the experiment [3]. The formula for effective electrode area $S_{*}=\gamma d \pi R$ obtained from simple geometric relations is usually used in electrical engineering [4]. Here, $\gamma=\Delta E /\left(E_{0}-\Delta E\right)$ where $\Delta E$ is the permissible field deviation from maximum field at apex of the sphere. Nevertheless, the relevant value of $\gamma$ is not well-defined and depends on the particular liquid. The formula (14) for $S_{*}$ introduced in our work naturally depends on features of particular dielectrics through the function $\mu(E)$ and also generally on electric field $E_{0}$. In the particular case of powerlaw approximation (16), the value of effective area $S_{*}$ for hemispherical electrodes does not depend on electric field and depends on only one parameter of specific dielectric liquid $n$. This situation may be valid for specific dielectric liquids in some ranges of electric field strength.

4.2.1. Breakdown under constant DC test-voltage. In the simplest case of constant DC test-voltage, the analytical expressions for the integral of electric-field action $H(t)$ were derived from (18) for hemispherical electrodes

$$
H(t)=\frac{d \pi R}{(n-1)} \mu\left(E_{0}\right) t .
$$

The exponential distribution (6) of random variable $t_{\mathrm{S}}$ is valid for this case as well as for flat electrodes.

4.2.2. Breakdown under DC ramp test-voltage. In the case of DC ramp test-voltage $V(t)=k t$, the expressions (17) and (18) can be integrated easily and the analytical expressions for $H(t)$ were derived for flat electrodes

$$
H(t)=\frac{d S A E^{n+1}}{(n+1) k E_{1}^{n}},
$$

and for hemispherical electrodes

$$
H(t)=\frac{d^{2} \pi R A E_{0}^{n+1}}{\left(n^{2}-1\right) k E_{1}^{n}} .
$$




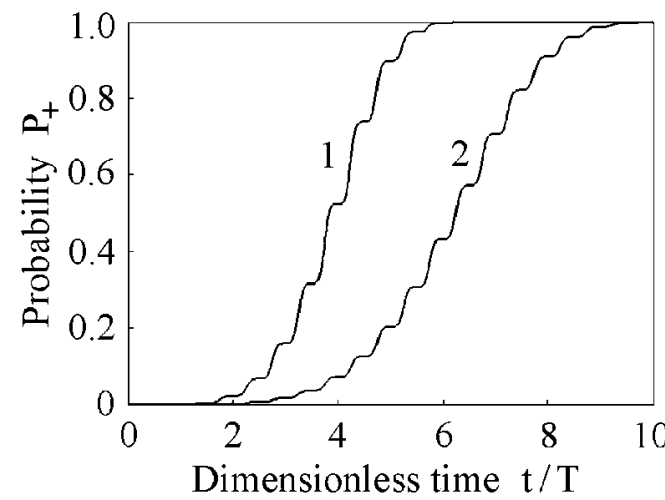

Figure 8. The probability of breakdown initiation for flat electrodes vs $t / T$, where $T$ is the period of AC voltage. $C=10^{-6}$ (curve 1 ), $10^{-7}$ (curve 2$), n=4$.

The effective value of time of electric-field action that takes into account the form of applied voltage and the form of power-law approximation of function $\mu(E)$ is $t_{*}=E d /(k(n+1))=t /(n+1)$.

\subsubsection{Breakdown under AC test-voltage of linearly increasing} amplitude. For AC voltage of linearly increasing amplitude, the current value of voltage is $V(t)=\sqrt{2} k_{\mathrm{e}} t \sin (\omega t)$ and, consequently, $E(t)=\sqrt{2} k_{\mathrm{e}} t \sin (\omega t) / d$ where $k_{\mathrm{e}}$ is the rate of increase of effective value of applied voltage. In the case of power-law approximation (16) of the function $\mu(E)$, one can obtain from (17) the expression for flat electrodes

$$
H(t)=C \int_{0}^{\omega t} z^{n}|\sin (z)|^{n} \mathrm{~d} z
$$

where $C=A S\left(\sqrt{2} k_{\mathrm{e}}\right)^{n}\left(E_{1}^{n} d^{n} \omega^{n+1}\right)$. The plot of $P_{+}$versus the dimensionless time $t / T$ is shown in figure 8 .

At AC voltage of slowly increasing amplitude, the product $k_{\mathrm{e}} t$ changes only slightly during each half-cycle and the form of every voltage pulse is practically proportional to $\sin (\omega t)$. In this case, the integral of electric-field action $H(t)$ changes over a half-cycle by the value

$$
\Delta H_{i}=\frac{S A E_{i}^{n} T}{2 \pi E_{1}^{n}} \int_{0}^{\pi} \sin ^{n}(z) \mathrm{d} z,
$$

where $E_{i}$ is the amplitude of the electric field $E=\sqrt{2} k_{\mathrm{e}} t / d$ when the number of voltage half-cycles is equal to $i$. Hence, in the case of AC voltage, the factor $(1 / \pi) \int_{0}^{\pi} \sin ^{n}(z) \mathrm{d} z$ come into the expressions for $H(t)$ [20-22] by analogy with the factor for effective power of AC voltage.

When breakdown occurs after many half-cycles of voltage, we have

$$
H(t)=\frac{S d A E^{n+1}}{\sqrt{2} k_{\mathrm{e}} \pi(n+1) E_{1}^{n}} \int_{0}^{\pi} \sin ^{n}(z) \mathrm{d} z .
$$

The effective value of time of electric-field action is

$t_{*}=\frac{E d}{\sqrt{2} k_{\mathrm{e}}(n+1) \pi} \int_{0}^{\pi} \sin ^{n}(z) \mathrm{d} z=\frac{t}{(n+1) \pi} \int_{0}^{\pi} \sin ^{n}(z) \mathrm{d} z$.

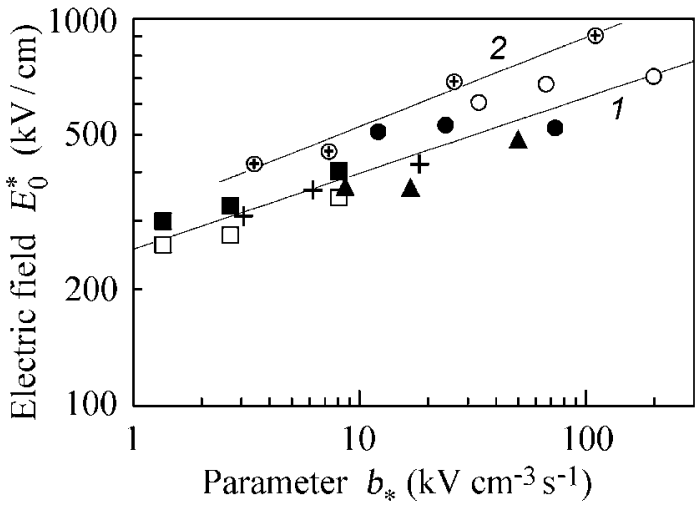

Figure 9. Dependence $E_{0}^{*}$ on parameter $b_{*}$. Curve 1 is for transformer oil. $d=0.5(\mathrm{O}), 0.83(\bullet), 1.0(\mathbf{\Delta}), 1.66(+), 2.5$ $(\square, \square) \mathrm{mm} . R=19 \mathrm{~mm}$. Curve 2 is for perfluorodibutyl ether $(\oplus)$. $d=0.44,0.9,1.7,2.5 \mathrm{~mm} . R=30 \mathrm{~mm}$.

Using approximations (3) and (11) we also obtained the following approximate formula for hemispherical electrodes with a small interelectrode gap:

$$
\Delta H_{i}=\frac{R d A E_{i 0}^{n} T}{2(n-1) E_{1}^{n}} \int_{0}^{\pi} \sin ^{n}(z) \mathrm{d} z .
$$

Here, $E_{i 0}$ is the amplitude of the average electric field on the axis between the electrodes. By analogy with (24)

$$
H(t)=\frac{R d^{2} A E_{0}^{n+1}}{\sqrt{2} k_{\mathrm{e}}\left(n^{2}-1\right) E_{1}^{n}} \int_{0}^{\pi} \sin ^{n}(z) \mathrm{d} z .
$$

Thus, from (4) and using the expressions for $H(t)$, one can obtain the dependences of the probability of breakdown on the main parameters such as the radius of the electrode surface (for hemispherical electrodes) or electrode area (in the case of flat electrodes), gap distance, rate of increase in voltage, etc. It is interesting, that the current value of electric strength $E$ corresponding to some fixed probability $P_{+}$depends only on parameter $b=k /(S d)$ for flat electrodes for DC ramp voltage. The effective value $k_{*}=\sqrt{2} k_{\mathrm{e}} \pi\left(\int_{0}^{\pi} \sin ^{n}(z) \mathrm{d} z\right)^{-1}$ should be used instead of $k$ for AC ramp voltage. For hemispherical electrodes, the effective area $S_{*}$ should be used instead of $S$. Nevertheless, specially for hemispherical electrodes and for AC ramp test-voltage, the parameter $b_{*}=k_{\mathrm{e}} /\left(\pi R d^{2}\right)$ is more convenient, because it does not depend on $n$. The parameters $b$ and $b_{*}$ could be used for preliminary comparing of the experimental data obtained at different geometry of electrodes, different values of $k_{\mathrm{e}}$, and $S$ (for flat electrodes) or $d$ and $R$ (for hemispherical electrodes). For example, the results of the experiments given in tables 1 and 2 were plotted as the dependences on the parameter $b_{*}$ (figure 9). One can conclude that the electrical strength of perfluorodibutyl ether is greater than that for transformer oil in wide range of values of parameter $b_{*}$.

\subsection{Reconstruction of $\mu(E)$ in the case of power-law approximation}

For test-voltage increasing in time, every value of voltage uniquely corresponds to the definite value of statistical time lag of breakdown initiation. In this case, several methods were 
developed to reconstruct the function $\mu(E)$ using experimental data (i) on histograms of breakdown voltages, (ii) on voltage corresponding to fixed breakdown probability; and (iii) on mean values of breakdown voltages.

4.3.1. Method of histograms of breakdown voltages. When large set of data on breakdowns in each series of experiments $\left(N_{0} \gg 100\right)$ is available, it is possible to reconstruct values of $\mu(E)$ using the histograms of breakdown voltages measured in experiments.

DC ramp test-voltage. From (20) and (21), we obtained the formulae, which expresses the values of $\mu(\mathrm{E})$ using the experimental distribution of breakdown voltages for flat electrodes

$$
\mu(E)=\frac{k \ln \left(N_{i} / N_{i+1}\right)}{S d \Delta E}
$$

and for hemispherical electrodes

$$
\mu(E)=\frac{k(n-1) \ln \left(N_{i} / N_{i+1}\right)}{\pi R d^{2} \Delta E} .
$$

Here $N_{i}$ and $N_{i+1}$ are the numbers of breakdowns in the series which occurred not earlier than the $i$ and $(i+1)$ interval of voltage, respectively, $\Delta E$ is the increment of the electric field strength at histogram.

AC test-voltage of linearly increasing amplitude. From (24), we obtained the formula that expresses the values of $\mu(E)$ using the experimental distribution of breakdown voltages for flat electrodes

$$
\mu(E)=\frac{\pi \sqrt{2} k_{\mathrm{e}} \ln \left(N_{i} / N_{i+l}\right)}{S d \Delta E_{l} \int_{0}^{\pi} \sin ^{n}(z) \mathrm{d} z} .
$$

Here $N_{i}$ and $N_{i+l}$ are the numbers of breakdowns in the series which occurred not earlier than the $i$ and $(i+l)$ voltage halfcycles, respectively, after the voltage was switched on, $\Delta E_{l}$ is the increment of the electric field strength over $l$ half-cycles (it was assumed that $l \ll i$ ).

From (27), it is easy to derive the formula for reconstruction of $\mu(E)$ from data on experimental distribution of breakdown voltages that is valid for hemispherical electrodes

$$
\mu(E)=\frac{\sqrt{2} k_{\mathrm{e}}(n-1) \ln \left(N_{i} / N_{i+l}\right)}{R d^{2} \Delta E_{l} \int_{0}^{\pi} \sin ^{n}(z) \mathrm{d} z} .
$$

The experimental data of Weber and Endicott [1] on breakdown in transformer oil under $\mathrm{AC}$ voltage with a frequency of $60 \mathrm{~Hz}$ were analysed using the method of histograms of breakdown voltages. In these experiments, four pairs of flat brass electrodes of areas $S=1.54,4.9,15$, and $29 \mathrm{~cm}^{2}$ were used at a gap spacing $d=0.19 \mathrm{~cm}$. The effective value of the applied voltage increased with a constant rate $k_{\mathrm{e}}=3 \mathrm{kV} \mathrm{s}^{-1}$. The results of reconstruction of the function $\mu(E)$ obtained from (30) for each pair of electrodes are shown in figure 10. The power-law approximation (16) of $\mu(E)$ $\left(E_{1}=1 \mathrm{MV} \mathrm{cm}^{-1}, n=12.1\right.$ and $\left.A=9.0 \times 10^{4} \mathrm{~cm}^{-2} \mathrm{~s}^{-1}\right)$ is shown by straight line in figure 10 and in figure 11 (straight line 4).

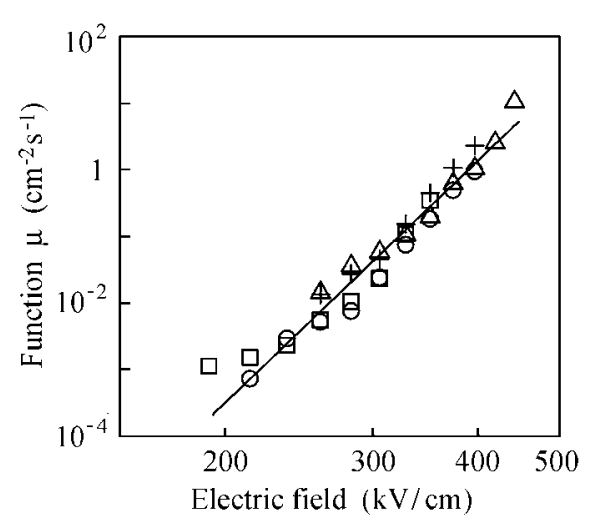

Figure 10. Reconstructed values of function $\mu(E)$ for transformer oil from the data of [1]. Pairs of flat brass electrodes of area $S=1.54(\triangle), 4.9(+), 15(\bigcirc), 29(\square) \mathrm{cm}^{2}$ were used. $N_{0}=400$ for each pair of electrodes.

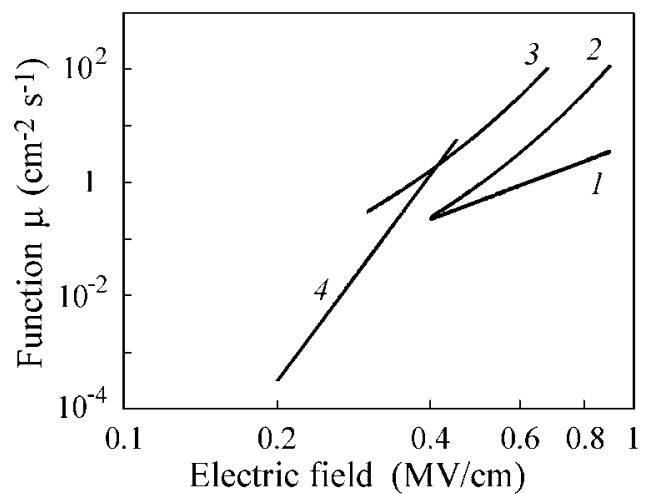

Figure 11. Values of function $\mu(E)$ reconstructed from experiment. Straight line 1 is the power-law approximation (16) of $\mu(E)$ for perfluorodibutyl ether. Curves 2 and 3 are approximations (47) of $\mu(E)$ for perfluorodibutyl ether and transformer oil, respectively. Straight line 4 is the function $\mu(E)$ reconstructed in [20-22] for transformer oil from the data of [1].

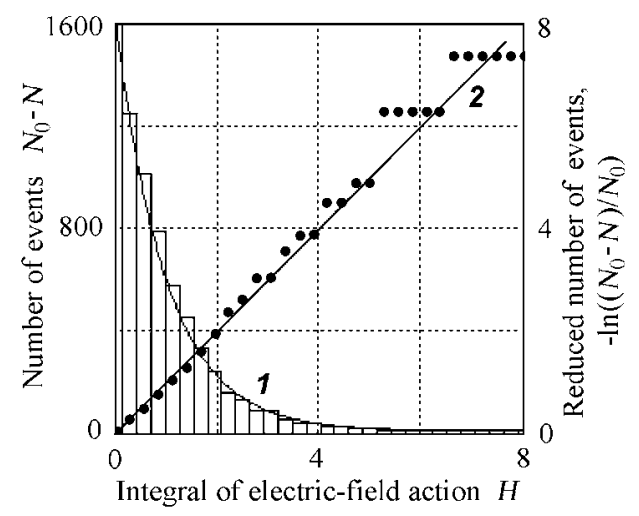

Figure 12. Bar chart of the distribution of $H$-values corresponding to the experimental data [1] on breakdowns in transformer oil. $N_{0}=1600$. Number of events $N_{0}-N$ is the number of breakdowns in the series which occurred after current value of $H(t)$.

Using expression (24) and the function $\mu(E)$ reconstructed for transformer oil, we calculated in [20] the fieldaction integrals $H$ for each of 1600 breakdowns presented in [1]. Figure 12 shows the distribution of these values of $H$. 
One can see that the bar chart agrees well with the exponential probability distribution $N_{0}-N=N_{0} \exp (-H)$ (curve 1). Indeed, the values $-\ln \left(\left(N_{0}-N\right) / N_{0}\right)$ versus $H$ are close to the straight line 2 that also confirms the theory proposed.

4.3.2. Method of fixed probability of breakdown. Another way to determine the parameters of function $\mu(E)$ from experiments is to use one of formulae (20), (21), (24) or (27) with fixed value of $H$ that correspond to some fixed probability of breakdown $P_{+}$. It is convenient to use the current values of electric field $E_{0}^{*}$ corresponding the value $H=1$, for which the probability of breakdown $P_{+}(t)=0.63$ [20-22].

DC ramp test-voltage. From (20) and (21) we obtained the explicit formulae that expresses the values of $\mu(E)$ at $E=E^{*}$ using the parameters of experiment and measured value of $E^{*}$ for flat electrodes

$$
\frac{\mu\left(E^{*}\right)}{(n+1)}=\frac{k}{S d E^{*}}
$$

and for hemispherical electrodes

$$
\frac{\mu\left(E_{0}^{*}\right)}{\left(n^{2}-1\right)}=\frac{k}{\pi d^{2} R E_{0}^{*}} .
$$

AC test-voltage of linearly increasing amplitude. By analogy, the formulae for reconstruction of $\mu(E)$ were obtained for AC test-voltage of linearly increasing amplitude for flat electrodes

$$
\frac{\mu\left(E^{*}\right)}{\pi(n+1)} \int_{0}^{\pi} \sin ^{n}(z) \mathrm{d} z=\frac{\sqrt{2} k_{\mathrm{e}}}{S d E^{*}}
$$

and for hemispherical electrodes

$$
\frac{\mu\left(E_{0}^{*}\right)}{\left(n^{2}-1\right)} \int_{0}^{\pi} \sin ^{n}(z) \mathrm{d} z=\frac{\sqrt{2} k_{\mathrm{e}}}{d^{2} R E_{0}^{*}} .
$$

The method of fixed probability could be applicable only for a large enough series of breakdowns $N_{0} \sim 100$. Otherwise, significant statistical variations of breakdown voltage (figure 1) result in some uncertainties in the values of $E^{*}$ and, consequently, in the reconstructed values of $\mu(E)$, because, the value of $E^{*}$ is determined mainly by local values of $E_{\mathrm{br}}$ in central part of the statistical distribution.

4.3.3. Method of mean values of an electric field of breakdown. At the same time, the mean value of amplitude of an electric field of breakdown $\left\langle E_{\mathrm{br}}\right\rangle$ could be determined from the same series of breakdown with smaller statistical error, because, in this case, the total information about $E_{\mathrm{br}}$ over the whole distribution is taken into account. In the case of powerlaw approximation (16) for $\mu(E)$, the explicit analytical expressions for mean value of current amplitude of electric field strength $\left\langle E_{\mathrm{br}}\right\rangle$, at which breakdown occurs, could be obtained from formula [5]

$$
\left\langle E_{\mathrm{br}}\right\rangle=\int_{0}^{\infty} E \frac{\mathrm{d} P_{+}}{\mathrm{d} E} \mathrm{~d} E,
$$

using the probability distribution (4) and (5).
DC ramp test-voltage. In the case of test-voltage $V(t)=k t$, we have for flat electrodes

$$
\left\langle E_{\mathrm{br}}\right\rangle=\left(\frac{k(n+1) E_{1}^{n}}{A S d}\right)^{1 /(n+1)} \Gamma\left(\frac{n+2}{n+1}\right)
$$

and for hemispherical electrodes

$$
\left\langle E_{0_{\mathrm{br}}}\right\rangle=\left(\frac{k\left(n^{2}-1\right) E_{1}^{n}}{A d^{2} R \pi}\right)^{1 /(n+1)} \Gamma\left(\frac{n+2}{n+1}\right) .
$$

From (37) and (38) the explicit expressions were obtained for reconstruction of values of function $\mu(E)$ at values of argument $E=\left\langle E_{\mathrm{br}}\right\rangle$.

For example, for flat electrodes, we have

$$
\mu\left(\left\langle E_{\mathrm{br}}\right\rangle\right) F(n)=\frac{k}{S d\left\langle E_{\mathrm{br}}\right\rangle},
$$

where the function

$$
F(n)=\frac{1}{(n+1) \Gamma((n+2) /(n+1))^{n+1}}
$$

depends only on the exponent $n$ in approximation (16) of $\mu(E)$.

For hemispherical electrodes, we have

$$
\mu\left(\left\langle E_{0_{\mathrm{br}}}\right\rangle\right) \frac{F(n)}{(n-1)}=\frac{k}{\pi R d^{2}\left\langle E_{0_{\mathrm{br}}}\right\rangle} .
$$

AC test-voltage of linearly increasing amplitude. Similar results were obtained also for AC test-voltage of linearly increasing amplitude. For flat electrodes, the following expression was obtained

$$
\left\langle E_{\mathrm{br}}\right\rangle^{n+1}=\frac{\sqrt{2} \pi(n+1) k_{\mathrm{e}} E_{1}^{n}}{A S d \int_{0}^{\pi} \sin ^{n}(z) \mathrm{d} z} \Gamma\left(\frac{n+2}{n+1}\right)^{n+1} .
$$

By analogy for hemispherical electrodes at small gap distance, the following formula is valid

$$
\left\langle E_{0_{\mathrm{br}}}\right\rangle^{n+1}=\frac{\sqrt{2}\left(n^{2}-1\right) k_{\mathrm{e}} E_{1}^{n}}{d^{2} R A \int_{0}^{\pi} \sin ^{n}(z) \mathrm{d} z} \Gamma\left(\frac{n+2}{n+1}\right)^{n+1} .
$$

One can see that the mean value of electric field of breakdown also depends on the parameters $b$ and $b_{*}$ mentioned above.

For AC test-voltage of linearly increasing amplitude, the explicit expressions were obtained from (42) and (43) for reconstruction of values of function $\mu(E)$ at values of argument $E=\left\langle E_{\mathrm{br}}\right\rangle$. For example, for flat electrodes, we have

$$
\mu\left(\left\langle E_{\mathrm{br}}\right\rangle\right) F_{\mathrm{AC}}(n)=\frac{\sqrt{2} k_{\mathrm{e}}}{S d\left\langle E_{\mathrm{br}}\right\rangle},
$$

where the function

$$
F_{\mathrm{AC}}(n)=\frac{\int_{0}^{\pi} \sin ^{n}(z) \mathrm{d} z}{\pi(n+1) \Gamma((n+2) /(n+1))^{n+1}}
$$

as well as (40) depends only on the exponent $n$ in approximation (16). The plot of function $F_{\mathrm{AC}}(n)$ is shown in figure 13. 


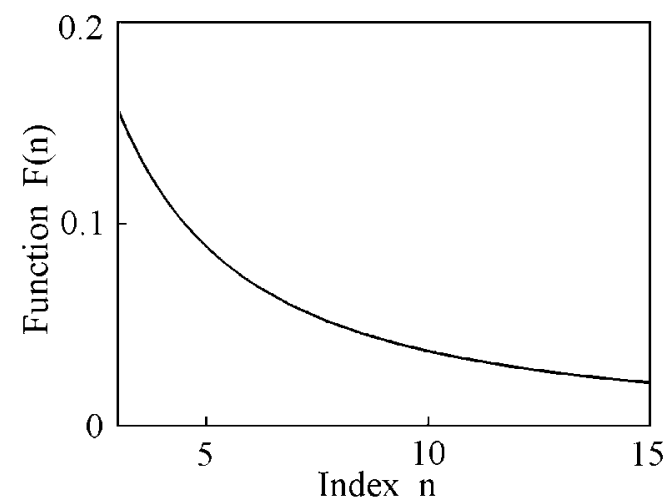

Figure 13. The dependence of function $F_{\mathrm{AC}}(n)$ on index $n$ in power-law approximation of $\mu(E)$.

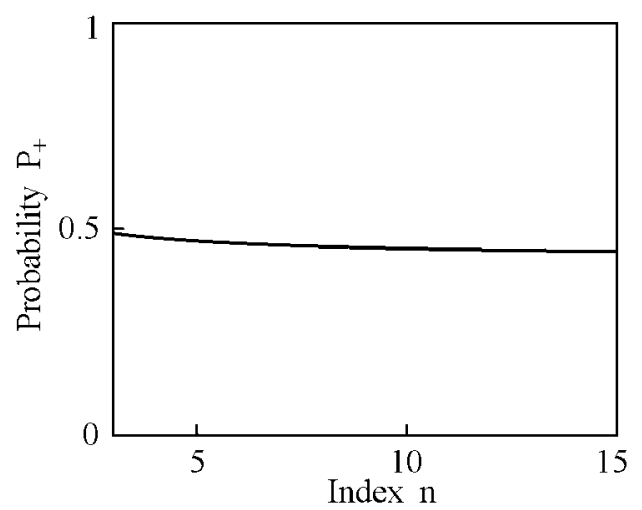

Figure 14. Function $P_{+}(n)$ corresponding the value $\left\langle E_{0_{\mathrm{br}}}\right\rangle$.

For hemispherical electrodes the explicit formula for reconstruction of values of function $\mu(E)$ was obtained using (43)

$$
\mu\left(\left\langle E_{0_{\mathrm{br}}}\right\rangle\right) \frac{F_{\mathrm{AC}}(n)}{n-1}=\frac{\sqrt{2} k_{\mathrm{e}}}{\pi d^{2} R\left\langle E_{0_{\mathrm{br}}}\right\rangle} .
$$

Obviously, the value of $\left\langle E_{\mathrm{br}}\right\rangle$ corresponds to some value of breakdown probability $P_{+}(n)$ depending on index $n$. The plot of corresponding values of probability $P_{+}(n)$ for hemispherical electrodes for AC test-voltage of linearly increasing amplitude is shown in figure 14. It is interesting, that the mean value of breakdown electric field $\left\langle E_{0_{\mathrm{br}}}\right\rangle$ corresponds in this case to a probability, that is only slightly less than $50 \%$ in the range of $n$ from 3 to 15 .

Thus, measuring the values of $\left\langle E_{\mathrm{br}}\right\rangle$ at different set of values $k$ or $k_{\mathrm{e}}$, and also $S$ or $(d$ and $R)$ depending on experimental conditions and using corresponding analytical expressions (39), (41), (44) or (46), one can reconstruct the function $\mu(E)$ from these experimental data.

For example, for AC test-voltage of linearly increasing amplitude and hemispherical electrodes at small gap distance using (46), we obtained the series of the values $\mu\left(\left\langle E_{0_{\mathrm{br}}}\right\rangle\right) F_{\mathrm{AC}}(n) /(n-1)$ at corresponding values of argument $E=\left\langle E_{0_{\mathrm{br}}}\right\rangle$ for experiments on breakdown in perfluorodibutyl ether for stainless steel electrodes (table 2). Using least squares fitting by straight line in $\log -\log$ scales, the value of index $n=3.39$ in approximation (16) was determined. Then, using the value of $F_{\mathrm{AC}}(n)$ calculated from (45), the absolute

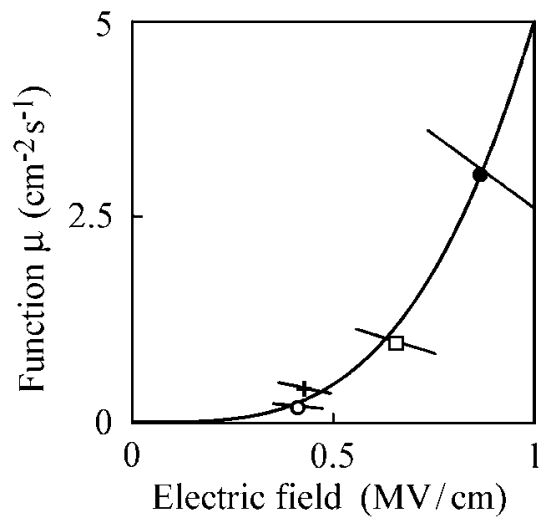

Figure 15. Power-law approximation of function $\mu(E)$ reconstructed for perfluorodibutyl ether. Gap distances between hemispherical stainless steel electrodes were $d=0.044,0.09,0.17$ and $0.25 \mathrm{~cm} . R=30 \mathrm{~mm}$.

values of function $\mu(E)$ and, hence, the value of parameter $A=5.0 \mathrm{~cm}^{-2} \mathrm{~s}^{-1}$ were determined in the range of variations of $\left\langle E_{0_{\mathrm{br}}}\right\rangle$ those took place in experiments. The values of function $\mu(E)$ are shown in figure 15 and 11 , curve 1 .

However, power-law approximation of function $\mu(E)$ gives too weak dependence on an electric field for perfluorodibutyl ether as shown in figure 11 (curve 1) that results in too wide scattering of breakdown voltage values in comparison with the experiments.

The results obtained from data of [1] for transformer oil using this method $\left(E_{1}=1 \mathrm{MV} \mathrm{cm}^{-1}, n=13.6\right.$ and $\left.A=4.5 \times 10^{5} \mathrm{~cm}^{-2} \mathrm{~s}^{-1}\right)$ are very close to the results obtained by the method of histograms.

\subsection{Special form of the approximation of $\mu(E)$}

The exponential function is monotonic too and it can be considered as an alternative approximation together with the simple power-law dependence. The calculations showed that the exponential approximation gives too sharp dependence on an electric field for perfluorodibutyl ether (too narrow scattering of breakdown voltage values in comparison with our experiments). Therefore, as an intermediate kind of approximation, we used a special form of the approximation of function $\mu(E)$

$$
\mu=A\left(\frac{E}{E_{1}}\right)^{2} \exp \left(\frac{E}{g}\right) .
$$

This function is more convenient because it allows one to calculate the integral over electric field in (12) and (14) analytically for hemispherical electrodes

$$
\begin{gathered}
H(t)=\frac{A d \pi R g}{E_{1}^{2}} \int_{0}^{t} E_{0}\left(\exp \left(\frac{E_{0}}{g}\right)-1\right) \mathrm{d} t, \\
S_{*}=\pi R d \frac{g}{E_{0}}\left(1-\exp \left(-\frac{E_{0}}{g}\right)\right) .
\end{gathered}
$$

The following calculations showed that special form of approximation of function $\mu(E)$ describes the histograms of breakdown voltages and breakdown pitting on a surface of hemispherical electrodes for our experiments better than power-law and exponential approximations. 
Usually $E_{0} \gg g$ and the effective area of hemispherical electrodes approximately is inversely proportional to the current value of electric field

$$
S_{*}=\pi R d \frac{g}{E_{0}} .
$$

Constant DC test-voltage. In this case, the explicit expressions (9) and (10) are valid again for flat electrodes. For hemispherical electrodes we have

$$
H(t)=\pi R d \frac{g}{E_{0}} A \frac{E_{0}^{2}}{E_{1}^{2}}\left(\exp \left(\frac{E_{0}}{g}\right)-1\right) t .
$$

For $E_{0} \gg g$, the approximate formula is valid

$$
H(t)=\pi R d \frac{g}{E_{0}} \mu\left(E_{0}\right) t .
$$

The equation for reconstruction of function $\mu(E)$ was obtained from (52)

$$
\mu\left(E_{0}\right) g=\frac{E_{0}}{\pi R d\left\langle t_{\mathrm{S}}\right\rangle} .
$$

More general approach that is valid for arbitrary form of function $\mu(E)$ was proposed in [17] and realized for hemispherical electrodes.

$D C$ ramp test-voltage. For test-voltage $V=k t$, the approximate expression for $H(t)$ was obtained in the case of $E_{0} \gg g$ for flat electrodes

$$
H(t)=\frac{S d g \mu(E)}{k} .
$$

For hemispherical electrodes, we have

$$
H(t)=\frac{\pi d^{2} R g^{2} \mu\left(E_{0}\right)}{k E_{0}} .
$$

The effective value of time of electric-field action is $t_{*}=$ $d g / k$ that takes into account the form of applied voltage and the special form of approximation (47) of function $\mu(E)$. Thus, in the non-power law form of $\mu(E)$, the effective value of time can be constant (does not depend on the current value of electric field and, consequently, on real time). This circumstance allows us to explain the different values of indexes in powerlaw approximations of time dependence and electrode surface dependence of breakdown voltage that is often experimentally obtained.

Using the method of fixed probability $(H=1)$, one can obtain the formula for reconstruction of function $\mu(E)$ from experimental data for flat electrodes

$$
\mu\left(E^{*}\right) g=\frac{k}{S d},
$$

and for hemispherical electrodes

$$
\mu\left(E_{0}^{*}\right) g^{2}=\frac{k E_{0}^{*}}{\pi d^{2} R} .
$$

AC test-voltage of linearly increasing amplitude. In the case of general form of time dependence of applied voltage, integration over time in (48) was carried out numerically for given values of $g$. For AC voltage of linearly increasing amplitude, this integration was carried out right up to the moment corresponding to the amplitude value of electric field $E_{0}^{*}$. Then, the parameter $A$ was obtained using the condition $H=1\left(P_{+}=0.63\right)$ for each series of breakdowns. Then, the pair of values of $g$ and $A$ was found that fitting the set of experimental data in the best way.

The values of function $\mu(E)$ reconstructed from experimental data on breakdowns in transformer oil (table 1) are shown in figure 11 (curve 3 ). The corresponding values of parameters in (47) are $E_{1}=1 \mathrm{MV} \mathrm{cm}^{-1}, g=0.09 \mathrm{MV} \mathrm{cm}^{-1}$ and $A=0.12 \mathrm{~cm}^{-2} \mathrm{~s}^{-1}$. The values of function $\mu(E)$ reconstructed from experimental data on breakdowns in perfluorodibutyl ether (table 2) are shown in figure 11 (curve 2). The corresponding values of parameters are $E_{1}=1 \mathrm{MV} \mathrm{cm}^{-1}$, $g=0.11 \mathrm{MV} \mathrm{cm}^{-1}$ and $A=0.04 \mathrm{~cm}^{-2} \mathrm{~s}^{-1}$.

\section{Stochastic simulation of breakdown initiation}

Using the reconstructed function $\mu(E)$, one can plot any dependencies of the breakdown initiation probability for various geometry of electrodes and also for various magnitude, duration and waveform of the applied voltage.

Within the framework of the stochastic approach proposed, computer simulation of series of breakdowns between hemispherical electrodes was carried out for AC test-voltage of linearly increasing amplitude using the special form (47) of the approximation of $\mu(E)$.

From (4) it follows that we can use random variable $H=-\ln (\zeta)$ in (48), where $\zeta$ is a random number that is uniformly distributed in the interval from 0 to 1 . Hence, the statistical time lag before breakdown $t_{\mathrm{S}}$ was determined from

$\int_{0}^{\omega t_{\mathrm{s}}} \tau|\sin \tau|[\exp (B \tau|\sin \tau|)-1] \mathrm{d} \tau=-\frac{\omega^{2} E_{1}^{2}}{\sqrt{2} \pi A g k_{\mathrm{e}} R} \ln (\zeta)$,

where $B=\sqrt{2} k_{\mathrm{e}} /(\omega d g)$. The right-hand side of (58) is a random value. Therefore, the integration on the left-hand side of (58) was carried out numerically over time until the value of the integral was equal to the value of expression on the right-hand side. The corresponding random value of current voltage $V\left(t_{\mathrm{S}}\right)$ at the breakdown and the current effective value of AC voltage $V_{\mathrm{EFF}}$ were calculated using the current value of the statistical time lag obtained. Then, using (11) and another random number $\zeta$, the random value of electric field $E$ at the surface of electrode was calculated from equation

$$
d \pi R E_{0} \int_{E}^{E_{0}} \frac{\mu(E)}{E^{2}} d E=\zeta d \pi R E_{0} \int_{0}^{E_{0}} \frac{\mu(E)}{E^{2}} \mathrm{~d} E .
$$

Hence, for special form of the approximation (47), the random value of electric field $E$ is equal to

$$
E=g \ln \left(\exp \left(\frac{E_{0}}{g}\right)-\zeta\left(\exp \left(\frac{E_{0}}{g}\right)-1\right)\right),
$$

where $E_{0}=V / d$. Then, the corresponding random value of polar angle $\theta$ on the surface of hemispherical electrode was calculated using the expression

$$
\cos \theta=1-\beta\left(\frac{E_{0}}{E}-1\right) .
$$

obtained from (3). 
The random value of azimuthal angle $\alpha=2 \pi \zeta$ was uniformly distributed in the interval from 0 to $2 \pi$. Thus, the random place of breakdown inception on the surface of hemispherical electrodes was determined

$$
x=R \sin \theta \cos \alpha, \quad y=R \sin \theta \sin \alpha .
$$

The product $a(\beta) E_{0}$ should be used instead of $E_{0}$ in all these equations for gaps that are not very narrow, where the correction factor mentioned above is equal to $a=1+0.666 \beta$.

Two typical series of breakdowns in transformer oil obtained in computer simulations are shown in figure 16 . These results are in good agreement with the experimental ones (figure 1).

Typical series of breakdowns in perfluorodibutyl ether obtained in computer simulations is shown in figure $3(b)$. This is in good agreement with experimental results (figure 3(a)). The results of simulations of breakdown pitting on the surface of hemispherical electrodes are shown in figure $17(a)$. This is also in reasonable agreement with experimental results (figure 17(b)).

Maximal difference $\Delta$ between values of $\left\langle V_{\mathrm{EFF}}\right\rangle$ obtained in experiments and in modelling is not greater than $20 \%$ for transformer oil (table 4) and is not greater than $16 \%$ for perfluorodibutyl ether (table 5). For most of series of breakdowns these differences are of order of standard deviations $\sigma$ observed in experiments.

The values of mean electric field of breakdown obtained in computer simulations are shown in figure 18(a) for transformer oil and in figure $18(b)$ for perfluorodibutyl ether. These values are in reasonable agreement with the experimental results.

(a)

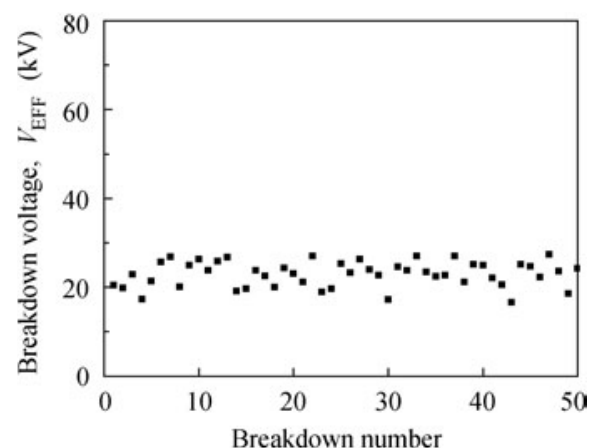

(b)

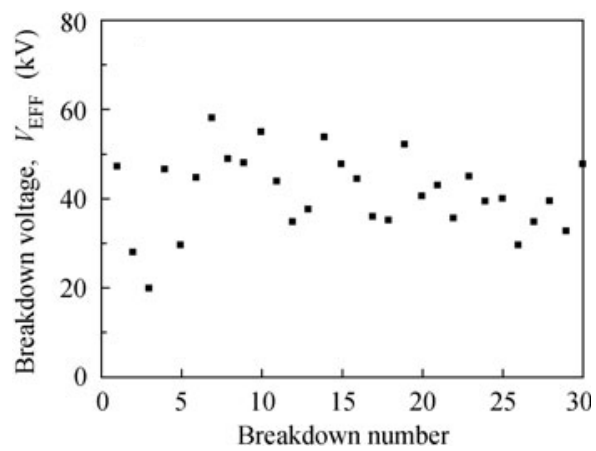

Figure 16. Computer simulation of series of breakdowns. (a) $d=0.5 \mathrm{~mm}, k_{\mathrm{e}}=3 \mathrm{kV} \mathrm{s}^{-1}$. (b) $d=1.66 \mathrm{~mm}, k_{\mathrm{e}}=1 \mathrm{kV} \mathrm{s}^{-1}$.

\section{Discussion and conclusions}

Our macroscopic approach is valid at least for small gaps (of the order of millimetres or less) where breakdown initiation is governed by area effect. Moreover, it should be noted that the formula (3) for approximate calculation of electric-field distribution along the surface of hemispherical electrodes is applicable for the values of reduced gap lengths $\beta \leqslant 0.1$. Hence, all the analytical formulae obtained for hemispherical electrodes are valid for breakdowns in only small gaps.

Only certain effective electric field dependences of functions $\mu(E)$ were actually found in the present work, because of the influence of electrode roughness [2, 5, 12,27]. For each dielectric liquid, all experiments were carried out with the same nominal roughness. Hence, the obtained values of $\mu(E)$ are valid only for fixed electrode roughness. An increase of nominal roughness $\delta$ by tenfold reduces the electrical strength of liquid helium and liquid nitrogen approximately down to $50 \%[5,12,27]$. These data can be fitted by the formula $\left\langle E_{\mathrm{br}}\right\rangle \sim \delta^{-v}$ [27] for $\delta$ being in certain range of order of 1 to $100 \mu \mathrm{m}$. One of the simple way to take into account the roughness of electrode is to introduce a factor $B(\delta)=\left(\delta / \delta_{0}\right)^{m}$ into the expression for $H(t)$

$$
H(t)=\int_{0}^{t}\left(\int_{S} B(\delta) \mu(E) \mathrm{d} s\right) \mathrm{d} t .
$$

Here, $m$ is the index that can be obtained from experiments.

The effect of polarity of DC voltage is related with the different mechanisms of breakdown inception on positive and negative electrodes. Prebreakdown processes are caused by thermal, bubble-like, ionization and others mechanisms, and begin at the anode or cathode, depending on pressure, temperature and electric field. For example, in $[18,19]$ the breakdown of $n$-hexane in strong electric fields $\left(E_{0}>1 \mathrm{MV} \mathrm{cm}^{-1}\right)$ in the time range from 10 to $1000 \mathrm{~ns}$ was investigated. At normal pressure, the competition of several mechanisms of breakdown inception took place (bubble-like mechanisms of breakdown inception both at the cathode and at the anode and ionization mechanism of breakdown inception at the anode). In this case, the integral of electric-field action depends on the mix of these mechanisms

$$
H(t)=\int_{0}^{t}\left(\int_{S_{+}} \mu_{+}(E) \mathrm{d} s+\int_{S_{-}} \mu_{-}(E) \mathrm{d} s\right) \mathrm{d} t,
$$

where $\mu_{+}(E)$ and $\mu_{-}(E)$ are the probability density functions of breakdown inception on a small element of area of positive and negative electrodes.

Experiments [18,19,28-31] point to the important role of gas phase on the stage of breakdown inception. The question about origin of micro-bubbles (pre-existing, thermally induced, electrically induced, etc) is still open, except for some well-defined experimental conditions [28]. In the range of fields where several independent mechanisms are operative their influence is additive. For example, we can obviously write $\mu(E)=\mu_{i}(E)+\mu_{b}(E)$ for ionization and bubble mechanisms. As the pressure increased to $\sim 1 \mathrm{MPa}$, the ionization mechanism for breakdown in $n$-hexane became 

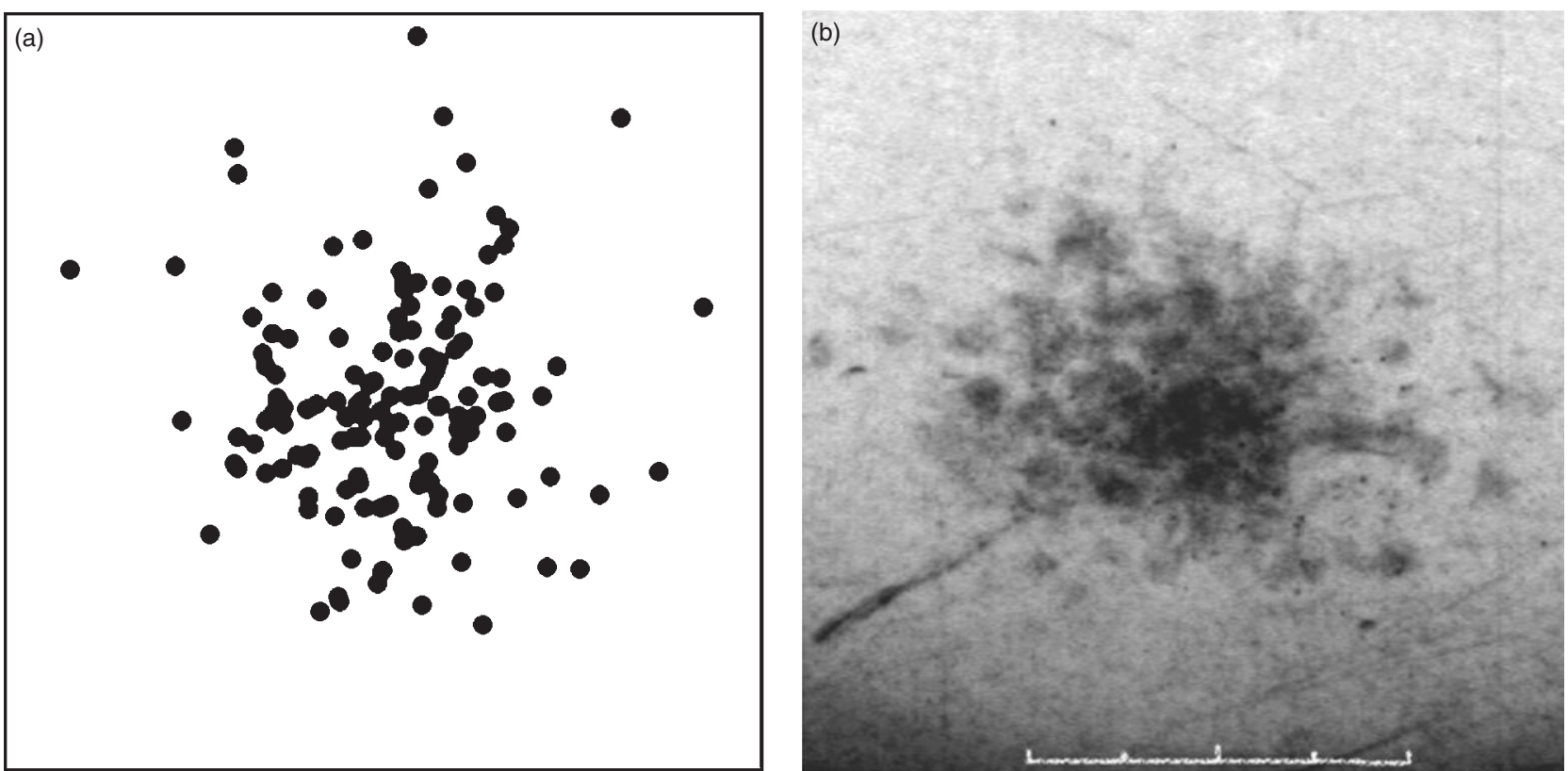

Figure 17. Breakdown pitting on surface of a hemispherical electrode. (a) Results of computer simulation of a series of breakdowns in perfluorodibutyl ether using approximation (47) at $g=0.11 \mathrm{MV} \mathrm{cm}^{-1}$ and $A=0.04 \mathrm{~cm}^{-2} \mathrm{~s}^{-1}$. (b) Photo of the electrode surface of stainless steel after a series of breakdowns in perfluorodibutyl ether. Areas of size $8 \times 8 \mathrm{~mm}$ are shown. $R=30 \mathrm{~mm}, d=0.44 \mathrm{~mm}, N_{0}=140$.

Table 4. Comparison of simulations and experiments on breakdown in transformer oil.

\begin{tabular}{|c|c|c|c|c|c|c|c|c|c|}
\hline \multirow[b]{2}{*}{ No } & \multirow[b]{2}{*}{$\begin{array}{l}d \\
(\mathrm{~mm})\end{array}$} & \multirow[b]{2}{*}{$\begin{array}{l}k_{\mathrm{e}} \\
\left(\mathrm{kV} \mathrm{s}^{-1}\right)\end{array}$} & \multicolumn{3}{|c|}{ Experiments } & \multicolumn{3}{|c|}{ Simulations, $N_{0}=130$} & \multirow[b]{2}{*}{$\begin{array}{l}\Delta \\
(\%)\end{array}$} \\
\hline & & & $\begin{array}{l}\left\langle V_{\mathrm{EFF}}\right\rangle \\
(\mathrm{kV})\end{array}$ & $\begin{array}{l}\sigma \\
(\mathrm{kV})\end{array}$ & $\begin{array}{l}\left\langle E_{0}\right\rangle \\
\left(\mathrm{kV} \mathrm{cm}^{-1}\right)\end{array}$ & $\begin{array}{l}\left\langle V_{\mathrm{EFF}}\right\rangle \\
(\mathrm{kV})\end{array}$ & $\begin{array}{l}\sigma \\
(\mathrm{kV})\end{array}$ & $\begin{array}{l}\left\langle E_{0}\right\rangle \\
\left(\mathrm{kV} \mathrm{cm}^{-1}\right)\end{array}$ & \\
\hline \multirow[t]{3}{*}{1} & 2.5 & 0.5 & 50.6 & 10.7 & 286 & 46.2 & 10.8 & 261 & -10 \\
\hline & 2.5 & 1 & 55.5 & 10.8 & 314 & 52.2 & 12.1 & 295 & -5 \\
\hline & 2.5 & 3 & 64.0 & 14.7 & 362 & 64.9 & 15.1 & 367 & 1 \\
\hline \multirow[t]{3}{*}{2} & 1.0 & 0.5 & 24.1 & 6.7 & 341 & 26.6 & 5.7 & 376 & 9 \\
\hline & 1.0 & 1 & 24.6 & 7.0 & 348 & 29.5 & 5.7 & 417 & 17 \\
\hline & 1.0 & 3 & 29.8 & 8.5 & 421 & 35.9 & 6.3 & 508 & 17 \\
\hline \multirow[t]{3}{*}{3} & 0.5 & 0.5 & 20.5 & 4.2 & 579 & 17.2 & 3.4 & 486 & -19 \\
\hline & 0.5 & 1 & 22.4 & 4.1 & 634 & 18.7 & 3.6 & 530 & -20 \\
\hline & 0.5 & 3 & 23.8 & 3.7 & 673 & 22.1 & 3.0 & 625 & -8 \\
\hline \multirow[t]{3}{*}{4} & 0.83 & 0.5 & 25.7 & 6.7 & 438 & 23.3 & 4.8 & 397 & -10 \\
\hline & 0.83 & 1 & 29.1 & 5.3 & 496 & 26.2 & 6.0 & 446 & -11 \\
\hline & 0.83 & 3 & 27.6 & 7.5 & 470 & 30.6 & 6.1 & 521 & 10 \\
\hline \multirow[t]{3}{*}{5} & 1.66 & 0.5 & 32.6 & 9.5 & 278 & 37.0 & 8.5 & 315 & 12 \\
\hline & 1.66 & 1 & 38.9 & 8.2 & 331 & 41.6 & 8.2 & 354 & 6 \\
\hline & 1.66 & 3 & 45.8 & 8.8 & 390 & 49.3 & 10.8 & 420 & 7 \\
\hline \multirow[t]{3}{*}{6} & 2.5 & 0.5 & 42.2 & 10.0 & 239 & 46.3 & 12.0 & 262 & 9 \\
\hline & 2.5 & 1 & 46.7 & 9.5 & 264 & 50.5 & 13.5 & 286 & 8 \\
\hline & 2.5 & 3 & 57.0 & 14.4 & 322 & 64.5 & 13.6 & 365 & 12 \\
\hline
\end{tabular}

dominant because the bubble-like mechanism was suppressed $[18,19]$. In this case, one of the integrals in (64) is much lesser than another one and may be neglected.

In the case of AC test-voltage, it is difficult to separate the mechanisms of breakdown inception. Specially for this case, we introduced the effective value of probability density function for identical electrodes $\mu(E)=\mu_{+}(E)+\mu_{-}(E)$. If the electrodes in pair are different, then the probability of breakdown inception should be described by

$$
H(t)=\int_{0}^{t}\left(\int_{S_{1}} \mu_{1}\left(E_{1}\right) \mathrm{d} s+\int_{S_{2}} \mu_{2}\left(E_{2}\right) \mathrm{d} s\right) \mathrm{d} t
$$

where $\mu_{1}\left(E_{1}\right)=\mu\left(E_{1}\right) / 2$ and $\mu_{2}\left(E_{2}\right)=\mu\left(E_{2}\right) / 2$ are the probability density functions of breakdown inception on a small element of one and another electrodes, respectively. For example, it was shown in [32] that the electrical strength of transformer oil for aluminium electrodes was greater by about $12 \%$ than that when using stainless steel electrodes.

If the material and roughness of both electrodes are the same, but the forms are different, the values and distributions of electric field on their surfaces will be different. For example, for long concentric coaxial electrodes, the values of electric field are constant along the surface of each electrode provided that edge effects are negligible. Hence, by analogy with [5], 
Table 5. Comparison of simulations and experiments on breakdown in perfluorodibutyl ether.

\begin{tabular}{|c|c|c|c|c|c|c|c|}
\hline \multirow[b]{2}{*}{$\begin{array}{l}d \\
(\mathrm{~mm})\end{array}$} & \multicolumn{3}{|c|}{ Experiments } & \multicolumn{3}{|c|}{ Simulations, $N_{0}=130$} & \multirow[b]{2}{*}{$\begin{array}{l}\Delta \\
(\%)\end{array}$} \\
\hline & $\begin{array}{l}\left\langle V_{\mathrm{EFF}}\right\rangle \\
(\mathrm{kV})\end{array}$ & $\begin{array}{l}\sigma \\
(\mathrm{kV})\end{array}$ & $\begin{array}{l}\left\langle E_{0}\right\rangle \\
\left(\mathrm{kV} \mathrm{cm}^{-1}\right)\end{array}$ & $\begin{array}{l}\left\langle V_{\mathrm{EFF}}\right\rangle \\
(\mathrm{kV})\end{array}$ & $\begin{array}{l}\sigma \\
(\mathrm{kV})\end{array}$ & $\begin{array}{l}\left\langle E_{0}\right\rangle \\
\left(\mathrm{kV} \mathrm{cm}^{-1}\right)\end{array}$ & \\
\hline \multicolumn{8}{|c|}{ Stainless steel electrodes, $R=30 \mathrm{~mm}$} \\
\hline 0.44 & 26.9 & 3.3 & 865 & 23.2 & 3.7 & 745 & -16 \\
\hline 0.9 & 41.2 & 7.3 & 647 & 38.1 & 8.0 & 599 & -8 \\
\hline 1.7 & 50.5 & 9.6 & 420 & 58.5 & 12.8 & 487 & 14 \\
\hline 2.5 & 70.8 & 11.8 & 400 & 73.3 & 19.2 & 415 & 4 \\
\hline \multicolumn{8}{|c|}{ Brass electrodes, $R=40 \mathrm{~mm}$} \\
\hline 0.44 & 20.3 & 5.0 & 652 & 22.5 & 3.7 & 722 & 10 \\
\hline 0.9 & 37.7 & 9.1 & 592 & 36.0 & 7.7 & 565 & -5 \\
\hline 1.7 & 49.4 & 6.4 & 411 & 55.1 & 12.2 & 459 & 10 \\
\hline 2.5 & 73.4 & 9.5 & 415 & 70.2 & 16.9 & 397 & -5 \\
\hline
\end{tabular}

(a)

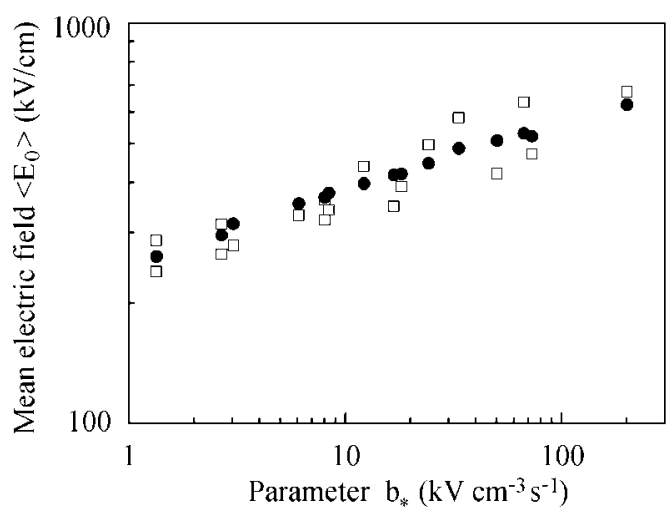

(b)

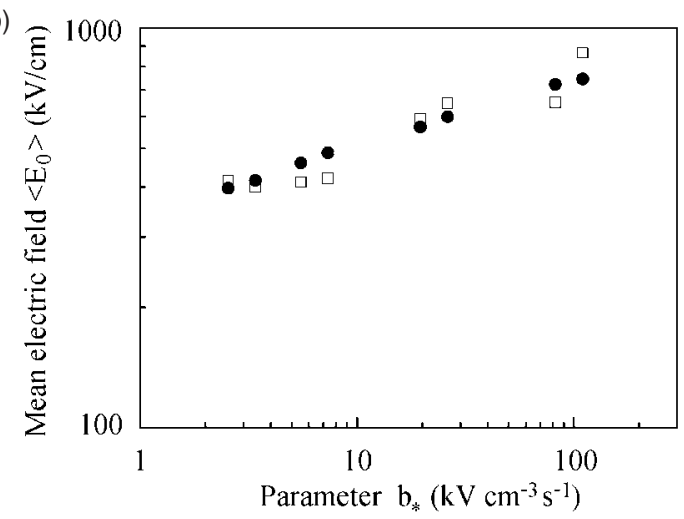

Figure 18. Experimental data ( $\square$ ) on breakdown in transformer oil $(a)$ and in perfluorodibutyl ether $(b)$ and computer simulations (๑) using approximation (47) at $g=0.09 \mathrm{MV} \mathrm{cm}^{-1}$ and $A=0.12 \mathrm{~cm}^{-2} \mathrm{~s}^{-1}(a)$, and at $g=0.11 \mathrm{MV} \mathrm{cm}^{-1}$ and $A=0.04 \mathrm{~cm}^{-2} \mathrm{~s}^{-1}(b)$.

we have

$$
H(t)=2 \pi h \int_{0}^{t}\left(a \mu_{1}(E)+b \mu_{1}\left(\frac{E a}{b}\right)\right) \mathrm{d} t,
$$

where $a$ and $b$ are the radii of inner and outer electrodes, $h$ is the length of work area of the electrodes, $E=V /(a \ln (b / a))$ is the value of electric field at the surface of inner electrode.

Within the framework of the proposed stochastic approach, several explicit dependences for probability distributions of breakdown initiation were analytically obtained for flat, hemispherical and coaxial cylindrical electrodes under DC constant voltage, DC ramp voltage and AC voltage of linearly increasing amplitude. This approach allows one to describe in standard way majority of phenomena of breakdown initiation in conditions when many parameters mentioned above simultaneously influence the electric strength of liquid dielectrics. It was found out that, our experimental data are described better using the supposition of the surface effect than of volume effect.

Usually the experimental data are fitted in $\log -\log$ scale by Weibull functions (depending on electric field, area of electrodes or time) [5-12]. In particular case, for powerlaw approximation of $\mu(E)$, we can obtain Weibull-like distributions from (4) and (17) for flat or from (18) for hemispherical electrodes. In this case, these distributions depend on two parameters in power-law approximation of function $\mu(E)$ on electric field. Hence, the Weibull distributions in time, size, and electric field strength in some sense are close to the particular forms of our distribution. However, the probability distribution (4) with our definition of function $H(t)$ in forms (5) or (12) is more general.

It was shown that the effective area of hemispherical electrodes in the case of narrow gaps between them is proportional to the product of the radius of electrode to the gap length. The coefficient naturally depends on features of particular dielectric through the function $\mu(E)$ and in general case depends on magnitude of electric field.

For the first time, the opportunity of direct stochastic computer simulations of experiments on breakdown in dielectric liquids is demonstrated. The proposed approach describes essentially stochastic nature of breakdown that is necessary to take into account at designing electrotechnical devices in which liquid dielectrics are employed.

\section{Acknowledgments}

This work was supported in part by the Siberian Branch of the Russian Academy of Science (Grant No 47-2000) and also was supported in part by the grants of the NATO Science Fellowship Programs-2000, 2001 and 2002.

\section{References}

[1] Weber K H and Endicott H S 1956 Area effect and its extremal basis for the electric breakdown of transformer oil Trans. Am. Inst. Electr. Eng. 75 371-81

[2] Lewis T J and Ward B W 1962 A statistical interpretation of the electrical breakdown of liquid dielectrics Proc. Roy. Soc. A: Math. Phys. Sci. 269 109-24

[3] Gerhold J, Hubmann M and Telser E 1994 Gap size effect on liquid helium breakdown Cryogenics 34 579-86

[4] Gauster W F 1956 Über Oberflächeneffekte beim elektrischen Durchbruch von Flüssigkeiten Österreichisches Ingenieur-Archiv 10 160-7

[5] Goshima H, Hayakawa N, Hikita M, Okubo H and Uchida K 1995 Weibull statistical analysis of area and volume effects on the breakdown strength in liquid nitrogen IEEE Trans. Diel. Electr. Insul. 2 385-93

[6] Suehiro J, Ohno K, Takahashi T, Miyama M and Hara M 1996 Statistical characteristics of electrical breakdown in saturated superfluid helium Proc. 12th Int. Conf. on Conduction and Breakdown in Dielectric Liquids (Roma Italy) IEEE No. 96CH35981, pp 320-3 
[7] Hill R M and Dissado L A 1983 Theoretical basis for the statistics of dielectric breakdown J. Phys. C: Solid State Phys. 16 2145-56

[8] Dissado L A, Forthergill J C, Wolfe S V and Hill R M 1984 Weibull statistics in dielectric breakdown: theoretical basis, applications and implications IEEE Trans. Electr. Insul. 19 227-33

[9] Dissado L A 1990 Theoretical basis for the statistics of dielectric breakdown J. Phys. D: Appl. Phys. 23 1582-91

[10] Gerhold J, Hubmann M and Telser E 1996 About the size effect in LHe-breakdown Proc. 12th Int. Conf. on Conduction and Breakdown in Dielectric Liquids (Roma, Italy) IEEE No. 96CH35981, pp 324-8

[11] Gerhold J, Hubmann M and Telser E 1998 Breakdown probability and size effect in liquid helium IEEE Trans. Diel. Electr. Insul. 5 321-33

[12] Gerhold J 1999 Cryogenic liquids - a prospective insulation basis for future power equipment Proc. 13th Int. Conf. on Dielectric Liquids (Nara, Japan) IEEE No. 99CH36213, pp 365-71

[13] Vorob'ev V V, Kapitonov V A, Kruglyakov E P and Tsidulko Yu A 1980 Breakdown of water in a system with 'diffusion' electrodes Sov. Phys. Tech. Phys. 25 598-602 (Transl. Russian Zh. Tekh. Fiz. 50 993-9)

[14] Kupershtokh A L 1992 Fluctuation model of the breakdown of liquid dielectrics Sov. Tech. Phys. Lett. 18 647-9 (Transl. Russian Pis'ma Zh. Tekh. Fiz. 18 no 19 91-6)

[15] Klimkin V F and Kupershtokh A L 1993 Statistical lag time in fluctuation model of liquid dielectric breakdown and experimental results Proc. 11th Int. Conf. on Conduction and Breakdown in Dielectric Liquid (Baden-Dättwil, Switzerland) IEEE No. 93CH3204-6, pp 395-9

[16] Vainer B G and Kupershtokh A L 1998 Measurements of statistical lag time of breakdown in thin amorphous layers of $\mathrm{SiO}_{2}$ Conf. Record 1998 IEEE Int. Symp. on Electrical Insulation (Arlington, USA) IEEE No. 98CH36239, pp 169-72

[17] Kupershtokh A L and Karpov D I 1999 Stochastic features of initiation of liquid dielectric breakdown at small area of positive electrode Proc. 13th Int. Conf. on Dielectric Liquids (Nara, Japan) IEEE No. 99CH36213, pp 203-6

[18] Borodin V P and Klimkin V F 1988 Pressure influence on mechanisms of electric breakdown in n-hexane Sov. Tech Phys. Lett. 14 (Transl. Russian Pis'ma Zh. Tekh. Fiz. 14 802-5)

[19] Klimkin V F 1990 Mechanisms of electric breakdown in n-hexane in the nanosecond range Sov. Phys. Tech. Phys. 35 735-6 (Transl. Russian Zh. Tekh. Fiz. 60 161-3)

[20] Kupershtokh A L, Palchikov E I, Karpov D I and Ershov A P 2000 Probability density function of electrical breakdown initiation in dielectric liquids under AC and DC voltage Proc. 2nd Int. Workshop on Electrical Conduction, Convection, and Breakdown in Fluids (Grenoble, France) pp 91-4
[21] Kupershtokh A L, Palchikov E I, Karpov D I and Ershov A P 2000 Stochastic regularities of electrical breakdown initiation in dielectric liquids under AC Voltage Proc. 6th Int. Conf. on Modern Problems of Electrophysics and Electrohydrodynamics of Liquids (St. Petersburg, Russia) pp 175-8

[22] Kupershtokh A L, Vitellas I, Agoris D P, Karpov D I and Charalambakos V P 2001 Stochastic regularities of electrical breakdown initiation in transformer oil Proc. 36th Int. Conf. (Annual Meeting) of the IEEE Industry Application Society (Chicago, USA) IEEE No. 01CH37248C, vol 4, pp 2729-36

[23] Fenimore C, Stricklett K L, Yamashita H, Kawai H, Forster E O and Pompili M 1990 The inception and structure of prebreakdown streamers in perfluorinated polyethers Proc. 2nd Int. Workshop on Electrical Conductoin, Convection and breakdown in fluids (Grenoble, France) IEEE No. 90CH2812-6, pp 430-5

[24] Yamashita H, Forster E O and Pompili M 1993 Streamer formation in perfluoropolyether under impulse conditions IEEE Trans. Electr. Insul. 28 324-9

[25] Korobeynikov S M, Sarin S G, Furin G G and Lipunov N B 1996 HV DC electrical strength of perfluorotriethylamine Russ. J. Eng. Thermophys. $6347-58$

[26] Miyagi K, Wakimoto K, Sano T and Nakao Y 1999 Effect of bubbles on breakdown strengths of perfluorocarbon liquid and the liquid with dissolved $\mathrm{SF}_{6}$ Proc. 13rd Int. Conf. on Dielectric Liquids (Nara, Japan) IEEE No. 99CH36213, pp 525-8

[27] Gerhold J 1993 Liquid helium breakdown in terms of temperature and electrode roughness Proc. 11th Int. Conf. on Conduction and Breakdown in Dielectric Liquid (Baden-Dättwil, Switzerland) IEEE No. 93CH3204-6, pp 254-8

[28] Kattan R, Denat A and Bonifaci N 1991 Formation of vapor bubbles in non-polar liquids initiated by current pulses IEEE Trans. on Electr. Insulation 26 656-62

[29] Dumitrescu L, Lesaint O, Bonifaci N, Denat A and Notingher P 2000 Streamer inception in cyclohexane under impulse voltage Proc. 2nd Int. Workshop on Electrical Conduction, Convection and Breakdown in Fluids (Grenoble, France) IEEE No. 90CH2812-6, pp 107-10

[30] Aitken F, Jomni F and Denat A 1998 Bubble formation by a corona discharge in dielectric liquids Proc. 3rd Int. Symp. on Cavitation (Grenoble, France) pp 45-50

[31] Denat A, Jomni F, Aitken F and Bonifaci N 1999 Generation of bubbles in liquid argon and nitrogen in divergent electric fields Proc. 13th Int. Conf. on Dielectric Liquids (Nara, Japan) IEEE No. 99CH36213, pp 384-7

[32] El-Zeftawy L A, El-Kholy S M and Eish T D 1990 Impulse breakdown of transformer oil under uniform field Proc. 10th Int. Conf. on Dielectric Liquids (Grenoble, France) IEEE No. 90CH2812-6, pp 584-7 